\title{
LARGE DISPLACEMENT OF CROSSBEAM STRUCTURE THROUGH ENERGY METHOD
}

\author{
A. Mitra, P. Sahoo and K. Saha \\ Department of Mechanical Engineering, \\ Jadavpur University, Kolkata 700032, India \\ Phone: +91332414 6890, Fax: +913324146890 \\ E-mail: samik893@gmail.com,psjume@gmail.com, kashinathsaha@gmail.com
}

\begin{abstract}
This paper undertakes a geometric nonlinear large displacement static analysis of crossbeam structure. A crossbeam structure comprises two beams in contact with their longitudinal axes perpendicular to each other, used effectively in civil and mechanical engineering, marine and aerospace structures. The energy method forms the basis for the mathematical formulation and the governing set of equations are obtained using the principle of extremisation of the total energy of the system in its equilibrium state. To obtain the solution, an iterative procedure is developed based on the reaction force generated between the two beams of the system. The method is validated by experiment and simulation through ANSYS v11. Results are presented in terms of plots of reaction force and the displacement of the interaction point versus load in dimensional form and additionally, the deflected shapes of the crossbeam structure at static equilibrium condition, under a particular load, are provided. The static response of the system has been studied for variation of beam thickness, loading pattern and position of the supporting beam.
\end{abstract}

Keywords: Crossbeam, variational method, geometric nonlinearity.

\section{INTRODUCTION}

In structural mechanics applications, nonlinear system response can occur for two reasons: material nonlinearity and geometric nonlinearity. In the first case, the constituent material behaviour, i.e., stress-strain relationship is nonlinear, whereas in geometric nonlinearity, the strain-displacement relationship is nonlinear. In cases of nonlinear strain-displacement, the large transverse displacement in the system incorporates a stretching effect, which provides additional stiffening to the structure. This is of particular interest to designers because it helps them achieve designs that are more compact with considerable material and cost savings. Therefore, large displacement analysis of structural elements due to geometric nonlinearity has always generated immense interest among researchers.

One of the basic structural elements is a beam, which can be used separately or in association with other beams or plates, to satisfy different structural requirements, such as stiffness enhancement, light weight, low cost, material saving etc. Research studies carried out in the field of nonlinear beam analysis have been recorded in different review papers. Reddy (1979) presented different finite element formulations related to structural elements and their vibrations. Sathyamoorthy (1982a) reviewed the 
works on classical methods of nonlinear (geometric, material and other type of nonlinearities) beam analysis. The same author (Sathyamoorthy 1982b) also surveyed the developments on nonlinear beam analysis under static and dynamic conditions using the finite elements methods. Kapania and Raciti (1989) reviewed advances in the analysis of laminated structures (beams and plates) using shear deformation theories and finite elements methods and also on the buckling of such structures. More recently, Marur (2001) put forward a review work, which reported on the nonlinear vibration formulations of beams through different phases of development. Agarwal et al. (2006) studied the geometric nonlinear effects of the static and dynamic behaviour of beams made of isotropic, composite and functionally graded materials, using first order shear deformation theory (FSDT). The free vibration problem of a beam under a large static deflection was investigated by Cornil et al. (2007), using the nonlinear equations of motion. To obtain a solution, these equations were decomposed into a set of nonlinear differential equations for static deflection and a set of linear differential equations for the dynamic problem. Kong et al. (2008) obtained analytical solutions for the static and dynamic problems of Euler-Bernoulli beams at the micro level, based on strain gradient elasticity theory. Marur (2007) developed analytical models for static and dynamic analysis of thin-walled frames, representing automotive side structures, considering joint flexibility.

In the case of stiffened plates, research work has gone through different phases and the evolution process can be traced with the help of the review works of Mukhopadhyay and Mukherjee (1989), and Bedair (1998). Different researchers have carried out nonlinear analysis of stiffened plates using different techniques and methodologies. Sapountzakis and Katsikadelis (2000) investigated elastic deformation of ribbed plates subjected to static, transverse and in-plane loading, using the analog equation method to solve nonlinearly coupled equations. Koko and Olson (1991) developed a new numerical technique for large deflection elastoplastic analysis of stiffened plates using super finite elements. Bedair (1997) presented a methodology for the analysis of multi-stiffened plates under lateral loading, based on energy formulation and to achieve the solution, employed a sequential quadratic programming (SQP) technique. Sheikh and Mukhopadhyay (2000) performed geometric nonlinear analysis of stiffened plates utilising the spline finite strip method and von Karman nonlinear plate theory. Turvey and Salehi (2008) analysed the elastoplastic large deflection response of pressure loaded and discretely stiffened circular plates. Bruback and Hellesland (2008) studied the strength criterion, both in local and global bending, of stiffened plates under in-plane loading, using semi-analytical large deflection analysis. Wutzow and Paiva (2008) employed integral equations and the boundary element method (BEM) to perform a linear analysis of stiffened plates. Sapountzakis and Mokos (2008) presented a general solution for the analysis of plates stiffened by arbitrarily placed parallel beams of arbitrary doubly symmetric cross section with deformable connections subjected to an arbitrary loading. Fernandes (2009) also introduced a boundary element method (BEM) formulation based on Kirchhoff's hypothesis to perform a linear bending analysis of plates reinforced by beams. Deb Nath et al. (2010) obtained an analytical solution of elastic fields for a stiffened plate subjected to axial tension and pure bending, using an alternative displacement potential approach.

A vast amount of research has established that stiffeners have enormous influence on the strength and stability of plate structures. However, the effect of a 
stiffening beam element on another beam has received little attention. Therefore, the present paper analyses the static responses of a crossbeam structure under transverse loading through energy formulation. A crossbeam structure (Figure 1), which is basically two beams in contact with their longitudinal axes perpendicular to each other so as to form a 'cross', can effectively be used separately or with plate elements, to construct civil and mechanical engineering, marine and aerospace structures. This paper takes into account the geometric nonlinearity by considering the nonlinear straindisplacement relations and uses a direct substitution method with a relaxation parameter to solve the set of nonlinear equations. The displacement fields are solved through an iterative procedure, which is based on the reaction force between the two beams of the system and considers the difference between the displacements of the two beams at their contact point as the termination criterion. For validation purposes, an experiment is performed for a particular loading scenario. In addition, results from the present analyses are compared with results generated by the finite element-based commercial package ANSYS (ver. 11). The results are presented in terms of reaction force-load and displacement-load plots along with the deflected shapes of the structure under loading. The distribution of axial displacement and stretching force along the beams is also provided.

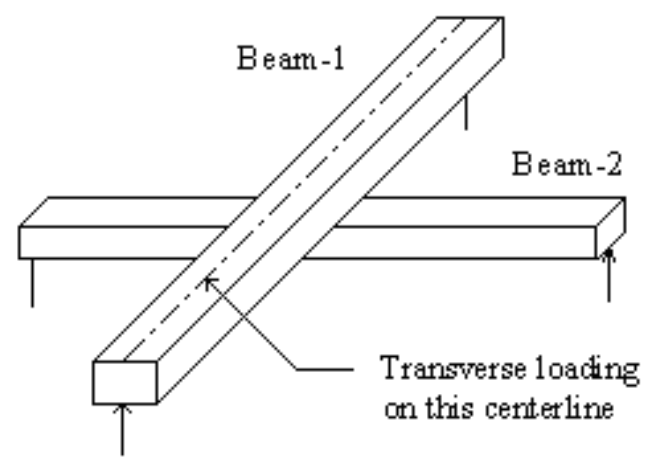

Figure 1. Crossbeam structure.

\begin{abstract}
ANALYSIS
The present paper analyses the large displacement static behaviour of a crossbeam structure consisting of two beams perpendicular to each other and placed in contact (Figure 1). At no loading condition, there is no interaction between the beams, although the beams are in surface contact. However, when some transverse load is applied to the upper beam (Beam-1), it pushes down on the lower beam (Beam-2), which in turn provides a resistance to deformation of Beam-1. It is assumed that external transverse loading acts on Beam-1 only and the purpose of Beam-2 is to give the structure a stiffening effect. The free body diagrams of the two beams are shown in Figure 2, along with the indications of nomenclatures of some system parameters. To maintain static equilibrium conditions for a particular loading, it can be said that:

$$
\begin{aligned}
& P+\int_{0}^{L_{1}} p(x) d x=R_{1}+R_{2}+R_{3}+R_{4}, \text { for the total system } \\
& P+\int_{0}^{L_{1}} p(x) d x-R=R_{1}+R_{2}, \text { for Beam- } 1 \\
& R=R_{3}+R_{4}, \text { for Beam- } 2
\end{aligned}
$$


where, $R$ is the unique reaction force generated at the contact between two beams. It is also implied that the displacement at the point of interaction of the two beams is equal.

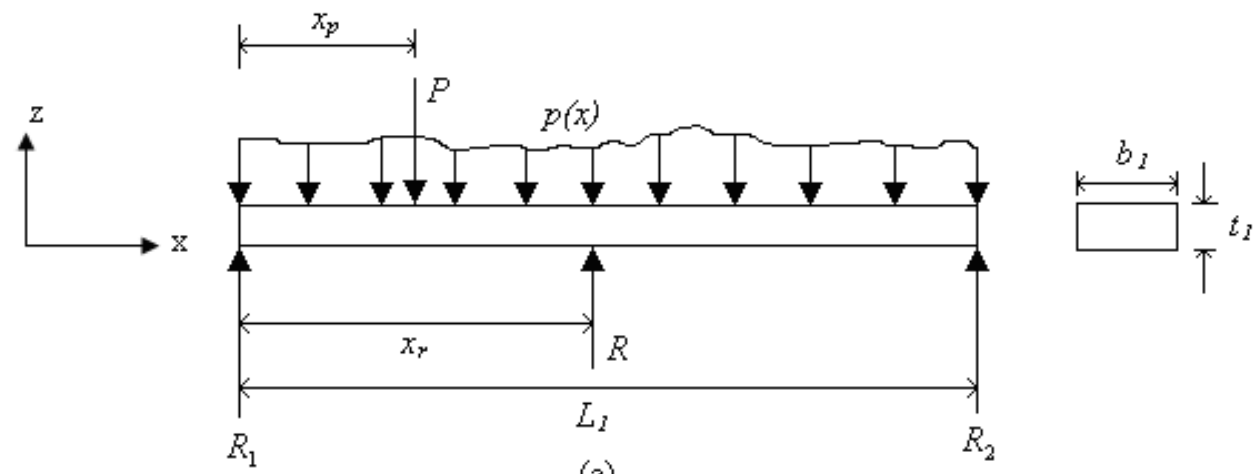

(a)

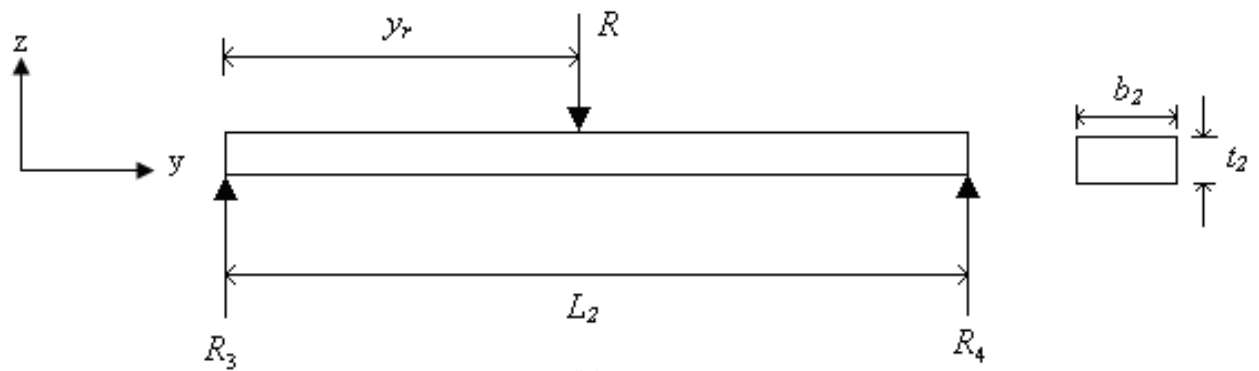

(b)

Figure 2. Free body diagrams of two individual beams: (a) Beam-1, (b) Beam-2.

\section{Mathematical Formulation}

The mathematical formulation of the individual beams is carried out through the energy method, which states that, $\delta(\pi)=0$, where $\pi=U+V$

$U=$ Total strain energy stored in the system

$V=$ Work function or potential of the external forces

$\delta=$ Variational operator

Furthermore, the formulation is based on the following assumptions:

i) Beam materials are isotropic, homogeneous and linearly elastic.

ii) Beams follow the Euler - Bernoulli hypothesis.

iii) Beams have uniform rectangular cross-section.

iv) The thicknesses of the beams are small compared to their respective lengths, such that the effect of shear deformation and rotary inertia are negligible.

In the case of large displacement analysis of beams, both bending and stretching effects are taken into consideration. Therefore, total strain energy stored in Beam- 1 is given by:

$U_{1}=U_{b 1}+U_{m 1}$

where:

$U_{b 1}=$ Strain energy stored due to bending $=\frac{1}{2} \int_{v o l} \sigma_{x} . \varepsilon_{x}^{b} d v$

$U_{m 1}=$ Strain energy stored due to stretching $=\frac{1}{2} \int_{v o l} \sigma_{x} \cdot \varepsilon_{x}^{s} d v$ 
$\varepsilon_{x}^{b}$ and $\varepsilon_{x}^{s}$ are axial strains due to bending and stretching, respectively. The axial strain due to bending at a distance $z$ from the mid-plane is given by: $\varepsilon_{x}^{b}=-z \frac{d^{2} w_{1}}{d x^{2}}$ and axial strain due to stretching of mid-plane is given by: $\varepsilon_{x}^{s}=\frac{d u_{1}}{d x}+\frac{1}{2}\left(\frac{d w_{1}}{d x}\right)^{2}$. Substituting these strain expressions into Eq. (3), the total strain energy stored in Beam-1 is:

$U_{1}=\frac{E_{1} I_{1}}{2} \int_{0}^{L_{1}}\left(\frac{d^{2} w_{1}}{d x^{2}}\right)^{2} d x+\frac{E_{1} A_{1}}{2} \int_{0}^{L_{1}}\left[\left(\frac{d u_{1}}{d x}\right)^{2}+\frac{1}{4}\left(\frac{d w_{1}}{d x}\right)^{4}+\left(\frac{d u_{1}}{d x}\right) \cdot\left(\frac{d w_{1}}{d x}\right)^{2}\right] d x$

Similarly, the total strain energy stored in Beam-2 is given by:

$U_{2}=\frac{E_{2} I_{2}}{2} \int_{0}^{L_{2}}\left(\frac{d^{2} w_{2}}{d y^{2}}\right)^{2} d y+\frac{E_{2} A_{2}}{2} \int_{0}^{L_{2}}\left[\left(\frac{d u_{2}}{d y}\right)^{2}+\frac{1}{4}\left(\frac{d w_{2}}{d y}\right)^{4}+\left(\frac{d u_{2}}{d y}\right) \cdot\left(\frac{d w_{2}}{d y}\right)^{2}\right] d y$

where: $w_{1}, w_{2}$ are transverse displacements of mid-plane of Beam-1 and Beam-2, respectively, $u_{1}, u_{2}$ are in-plane displacements of mid-plane of Beam-1 and Beam-2, respectively, $E_{1}, E_{2}$ are elastic moduli of Beam-1 and Beam-2, respectively, $L_{1}, L_{2}$ are lengths of Beam-1 and Beam-2, respectively, $x, y$ are axial coordinates in two orthogonal directions. $I_{1}, I_{2}$ and $A_{1}, A_{2}$ are second moment of area and cross sectional area of the two beams, respectively. The computations are carried out in normalised coordinates $\xi$ and $\eta$, respectively, where $\xi=x / L_{1}$ and $\eta=y / L_{2}$. The work potential of the external loads for Beam-1 and Beam-2 are given by:

$$
\begin{aligned}
& V_{1}=\left.P w_{1}\right|_{x_{p}}+\int_{0}^{L_{1}}\left(p w_{1}\right) d x-\left.R w_{1}\right|_{x_{r}} \\
& V_{2}=\left.R w_{2}\right|_{y_{r}}
\end{aligned}
$$

where: $P$ and $p$ represent the external concentrated and pressure type loading acting on Beam- 1 and $R$ is the reaction force generated between the two beams, as mentioned earlier. In addition, $x_{p}$ represents the point of application of the external concentrated load $(P)$ on Beam1, $x_{r}$ and $y_{r}$ denote the location, where the reaction force $(R)$ acts on Beam-1 and Beam-2, respectively. These notations are indicated in Figure 2.

The displacement functions $w_{1}, w_{2}$ and $u_{1}, u_{2}$ can be represented approximately by sets of orthogonal coordinate functions $\phi_{1 i}, \phi_{2 i}$ and $\alpha_{1 i}, \alpha_{2 i}$.

$$
\begin{aligned}
& w_{1}(\xi)=\sum_{i=1}^{n w} d_{1 i} \phi_{1 i}(\xi) \text { and } u_{1}(\xi)=\sum_{i=n w+1}^{n w+n u} d_{1 i} \alpha_{1 i-n w}(\xi) \\
& w_{2}(\eta)=\sum_{i=1}^{n w} d_{2 i} \phi_{2 i}(\eta) \text { and } u_{2}(\eta)=\sum_{i=n w+1}^{n w+n u} d_{2 i} \alpha_{2 i-n w}(\eta)
\end{aligned}
$$


where: $d_{1 i}$ and $d_{2 i}$ represent unknown coefficients and $n w$ and $n u$ are the number of functions for $w$ and $u$, respectively. Appropriate start functions for $w_{1}, u_{1}$ and $w_{2}, u_{2}$ are selected in such a way that they satisfy the necessary geometric boundary conditions of the respective beams. The higher order functions are generated from the selected start functions using the Gram-Schmidt orthogonalisation scheme.

Substituting Eq. (4), (6), (8) and (5), (7), (9) separately in Eq. (2) gives the governing set of equations for Beam-1 and Beam-2, respectively. For Beam-1, the governing set of equations in matrix form is given by: $\left[K^{1}\right]\left\{d_{1}\right\}=\left\{f_{1}\right\}$ where, $\left[K^{1}\right]$ and $\left\{f_{1}\right\}$ are the stiffness matrix and load vector, respectively and are of the following form: $\left[K^{1}\right]=\left[\begin{array}{ll}K_{11}^{1} & K_{12}^{1} \\ K_{21}^{1} & K_{22}^{1}\end{array}\right]$ and $\left\{f_{1}\right\}=\left\{\begin{array}{l}f_{11}^{1} \\ f_{12}^{1}\end{array}\right\}$. The elements of $\left\lfloor K^{1}\right\rfloor$ and $\left\{f_{1}\right\}$ are:

$$
\begin{aligned}
{\left[K_{11}^{1}\right]=} & \frac{E_{1} I_{1}}{L_{1}^{3}} \sum_{j=1}^{n w} \sum_{i=1}^{n w} \int_{0}^{1} \frac{d^{2} \phi_{1 i}}{d \xi^{2}} \frac{d^{2} \phi_{1 j}}{d \xi^{2}} d \xi+\frac{E_{1} A_{1}}{2 L_{1}^{3}} \sum_{j=1}^{n w} \sum_{i=1}^{n w} \int_{0}^{1}\left(\sum_{i=1}^{n w} d_{1 i} \frac{d \phi_{1 i}}{d \xi}\right)^{2} \frac{d \phi_{1 i}}{d \xi} \frac{d \phi_{1 j}}{d \xi} d \xi \\
& +\frac{E_{1} A_{1}}{L_{1}^{2}} \sum_{j=1}^{n w} \sum_{i=1}^{n w} \int_{0}^{1}\left(\sum_{i=n w+1}^{n w+n u} d_{1 i} \frac{d \alpha_{1 i-n w}}{d \xi}\right)^{2} \frac{d \phi_{1 i}}{d \xi} \frac{d \phi_{1 j}}{d \xi} d \xi \\
{\left[K_{12}^{1}\right]=} & 0,\left[K_{21}^{1}\right]=\frac{E_{1} A_{1}}{2 L_{1}^{2}} \sum_{j=n w+1}^{n w+n u} \sum_{i=1}^{n w} \int_{0}^{1}\left(\sum_{i=1}^{n w} d_{1 i} \frac{d \phi_{1 i}}{d \xi}\right) \frac{d \phi_{1 i}}{d \xi} \frac{d \alpha_{1 j-n w}}{d \xi} d \xi \\
{\left[K_{22}^{1}\right]=} & \frac{E_{1} A_{1}}{L_{1}} \sum_{j=n w+1}^{n w+n u} \sum_{i=n w+1}^{n w+n u} \int_{0}^{1} \frac{d \alpha_{1 i-n w}}{d \xi} \frac{d \alpha_{1 j-n w}}{d \xi} d \xi \\
\left\{f_{11}^{1}\right\}= & \left.\sum_{j=1}^{n w} P \phi_{1 j}\right|_{\xi}+L_{1} \sum_{j=1}^{n w} \int_{0}^{1} p(\xi) \phi_{1 j} d \xi-\left.\sum_{j=1}^{n w} R \phi_{1 j}\right|_{\xi_{r}},\left\{f_{12}^{1}\right\}=0
\end{aligned}
$$

Similarly, for Beam-2, the governing set of equations in matrix form is:

$$
\left[K^{2}\right]\left\{d_{2}\right\}=\left\{f_{2}\right\}
$$

where, $\left\lfloor K^{2}\right\rfloor$ and $\left\{f_{2}\right\}$ are the stiffness matrix and load vector, respectively and are of the following form: $\left[K^{2}\right]=\left[\begin{array}{cc}K_{11}^{2} & K_{12}^{2} \\ K_{21}^{2} & K_{22}^{2}\end{array}\right]$ and $\left\{f_{2}\right\}=\left\{\begin{array}{l}f_{11}^{2} \\ f_{12}^{2}\end{array}\right\}$. The elements of $\left\lfloor K^{2}\right\rfloor$ and $\left\{f_{2}\right\}$ are:

$$
\begin{aligned}
{\left[K_{11}^{2}\right]=} & \frac{E_{2} I_{2}}{L_{2}^{3}} \sum_{j=1}^{n w} \sum_{i=1}^{n w} \int_{0}^{1} \frac{d^{2} \phi_{2 i}}{d \eta^{2}} \frac{d^{2} \phi_{2 j}}{d \eta^{2}} d \eta+\frac{E_{2} A_{2}}{2 L_{2}^{3}} \sum_{j=1}^{n w} \sum_{i=1}^{n w} \int_{0}^{1}\left(\sum_{i=1}^{n w} d_{2 i} \frac{d \phi_{2 i}}{d \eta}\right)^{2} \frac{d \phi_{2 i}}{d \eta} \frac{d \phi_{2 j}}{d \eta} d \eta \\
& +\frac{E_{2} A_{2}}{L_{2}^{2}} \sum_{j=1}^{n w} \sum_{i=1}^{n w} \int_{0}^{1}\left(\sum_{i=n w+1}^{n w+n u} d_{2 i} \frac{d \alpha_{2 i-n w}}{d \eta}\right)^{2} \frac{d \phi_{2 i}}{d \eta} \frac{d \phi_{2 j}}{d \eta} d \eta
\end{aligned}
$$




$$
\begin{aligned}
& \left\lfloor K_{12}^{2}\right]=0,\left[K_{21}^{2}\right]=\frac{E_{2} A_{2}}{2 L_{2}^{2}} \sum_{j=n w+1}^{n w+n u} \sum_{i=1}^{n w} \int_{0}^{1}\left(\sum_{i=1}^{n w} d_{2 i} \frac{d \phi_{2 i}}{d \eta}\right) \frac{d \phi_{2 i}}{d \eta} \frac{d \alpha_{2 j-n w}}{d \eta} d \eta \\
& {\left[K_{22}^{2}\right]=\frac{E_{2} A_{2}}{L_{2}} \sum_{j=n w+1}^{n w+n u} \sum_{i=n w+1}^{n w+n n} \int_{0}^{1} \frac{d \alpha_{2 i-n w}}{d \eta} \frac{d \alpha_{2 j-n w}}{d \eta} d \eta} \\
& \left\{f_{11}^{2}\right\}=\left.\sum_{j=1}^{n w} R \phi_{2 j}\right|_{\eta_{r}},\left\{f_{12}^{2}\right\}=0
\end{aligned}
$$

\section{Solution Procedure}

The set of governing equations (Eqs (10) and (11)) are clearly nonlinear in nature, because the stiffness matrix itself is a function of unknown coefficients and solved by direct substitution technique using a successive relaxation scheme. For each load-step, the values of the unknown coefficients are assumed to evaluate the stiffness matrix. Using this stiffness matrix, based on the assumed values, new values of unknown coefficients are calculated by the matrix inversion technique from the expressions $\left\{d_{1}\right\}=\left[K^{1}\right]^{-1}\left\{f_{1}\right\}$ and $\left\{d_{2}\right\}=\left[K^{2}\right]^{-1}\left\{f_{2}\right\}$. Calculated values are compared with their values in the previous iteration and if the difference is above a predefined error limit, the process is repeated with new values of unknown coefficients and modified with a relaxation parameter, until the difference becomes less than the predefined error limit. When convergence is achieved for both beams, $\left\{d_{1}\right\}$ and $\left\{d_{2}\right\}$ are known and thus, from Eqs (8) and (9), the displacement fields for the two beams become apparent. This process can only be implemented if the load vectors $\left(\left\{f_{1}\right\}\right.$ and $\left.\left\{f_{2}\right\}\right)$ are known parameters. However, for a particular load applied to the crossbeam structure, the reaction force $(R)$ generated at the contact between two beams is not known beforehand. To overcome this hurdle an iterative process is devised, in which the reaction force is assumed to be a fraction $(q)$ of the total load applied to the structure, i.e., $R=q \times\left[\int_{0}^{1} p(\xi) d \xi+P\right]$. Using this assumed value, the load vectors are calculated and the set of unknown coefficients, as well as the displacement fields are solved.

Let the deflection of Beam-1 and Beam-2 at the point of contact be $\delta_{b 1}$ and $\delta_{b 2}$, respectively. If $\left(\delta_{b 1}-\delta_{b 2}\right) / t_{\min }$ [where, $\left.t_{\min }=\min \left(t_{1}, t_{2}\right)\right]$ is outside a permitted tolerance limit, the assumed reaction force is changed by modifying the fraction $(q)$ and using this new assumed reaction force, the process is repeated. When $\left(\delta_{b 1}-\delta_{b 2}\right) / t_{\text {min }}$ falls within the permissible value, the process is said to have converged and the unique reaction force and the displacement fields concerned with that particular load value is established. The modification of the assumed reaction force is carried out as follows: At the start, the correct value of $q\left(q_{\text {exact }}\right)$ is unknown but the minimum and maximum values of $q$ are known to lie between 0 and 1, i.e., $q_{\min }=0$ and $q_{\max }=1$. At the end of each iteration step, a part of the solution space can be eliminated depending on whether $\delta_{b 1}$ is greater or lower than $\delta_{b 2}$, thus changing $q_{\min }$ or $q_{\max }$. 
If $\delta_{b 1}>\delta_{b 2}: q$ needs to be increased in order to lower $\delta_{b 1}$ and increase $\delta_{b 2}$. This implies that $q_{\text {exact }}$ cannot be lower than the present $q$ and $q_{\text {min }}$ can be upgraded to this $q$ value. After modification of the limit, the new value $q$ is determined using the following expression, $q=q_{\min }+q_{1}\left(q_{\max }-q_{\min }\right)$, where $q_{1}$ is another load distribution parameter and $q_{1}$ lies between 0 and $1\left(0<q_{1}<1\right)$.

If $\delta_{b 1}<\delta_{b 2}: q$ needs to be decreased in order to increase $\delta_{b 1}$ and lower $\delta_{b 2}$. This implies that $q_{\text {exact }}$ cannot be greater than the present $q$ and $q_{\text {max }}$ can be upgraded to this $q$ value. After the modification of the limit, the new value $q$ is determined using the following expression, $q=q_{\max }-q_{1}\left(q_{\max }-q_{\min }\right)$, where $q_{1}$ is another load distribution parameter and $0<q_{1}<1$. The solution procedure is elaborated in the flow chart, shown in Figure 3.

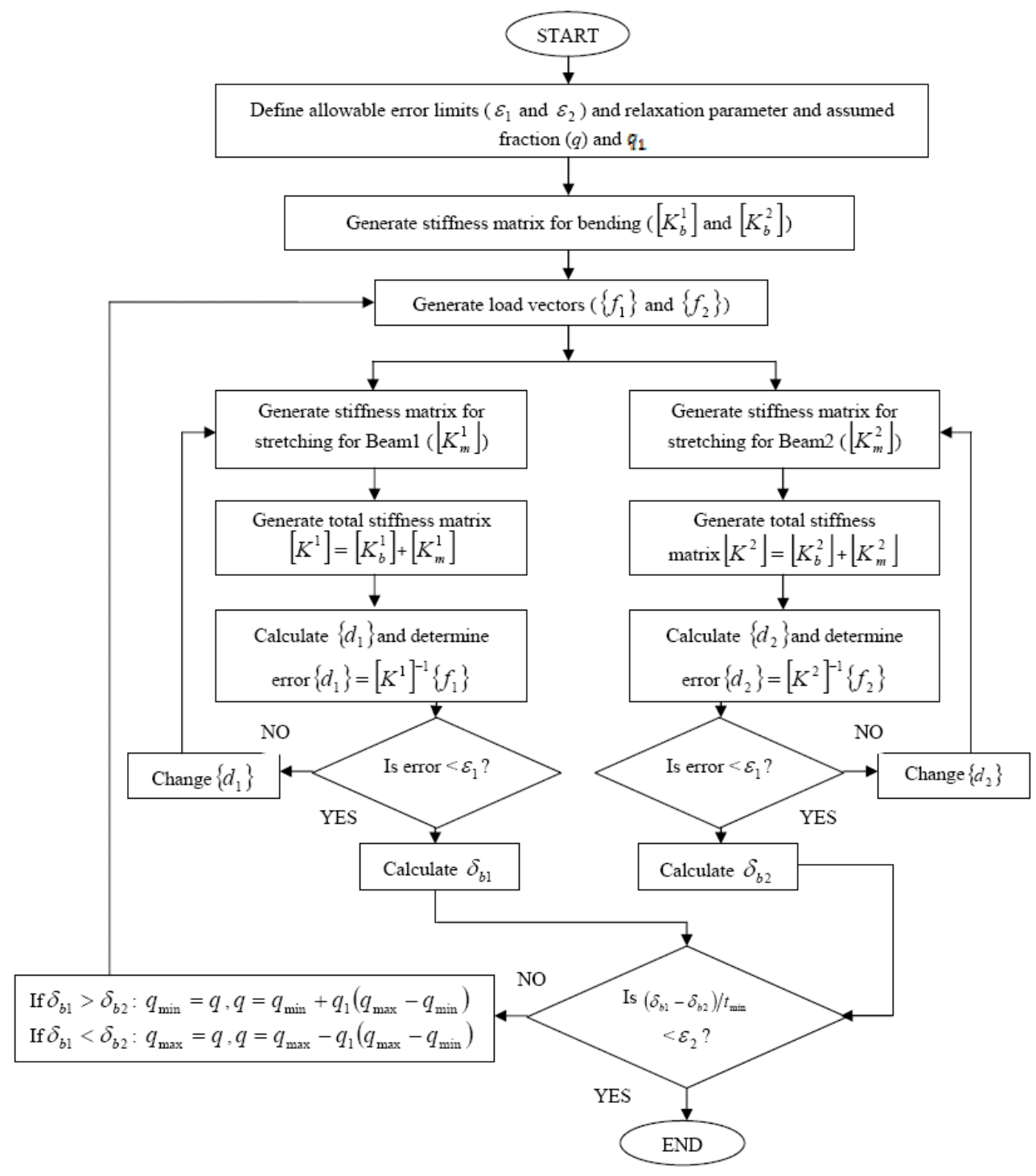

Figure 3. Flow chart of the solution algorithm. 
In the present paper, four different loading patterns are considered: concentrated load, uniformly distributed load, triangular load and hat load, as shown in Figure 4. However, for all these types of loading, boundary conditions of the beams have been considered as all ends are clamped (CC-CC). For membrane boundary conditions, it is assumed that in-plane displacements are zero at the boundaries, i.e., the boundaries are sufficiently rigid to support the axial load generated in the beams. In all the cases, the contact point is assumed to be at the mid-span of Beam-2, i.e., $y_{r}=0.5 L_{2}$. Consequently, the crossbeam structure can be equivalently represented by two individual beams; one carrying the external transverse loading along with the reaction force and the other only carrying the reaction force equal in magnitude and opposite in direction compared with the reaction force in the first beam.

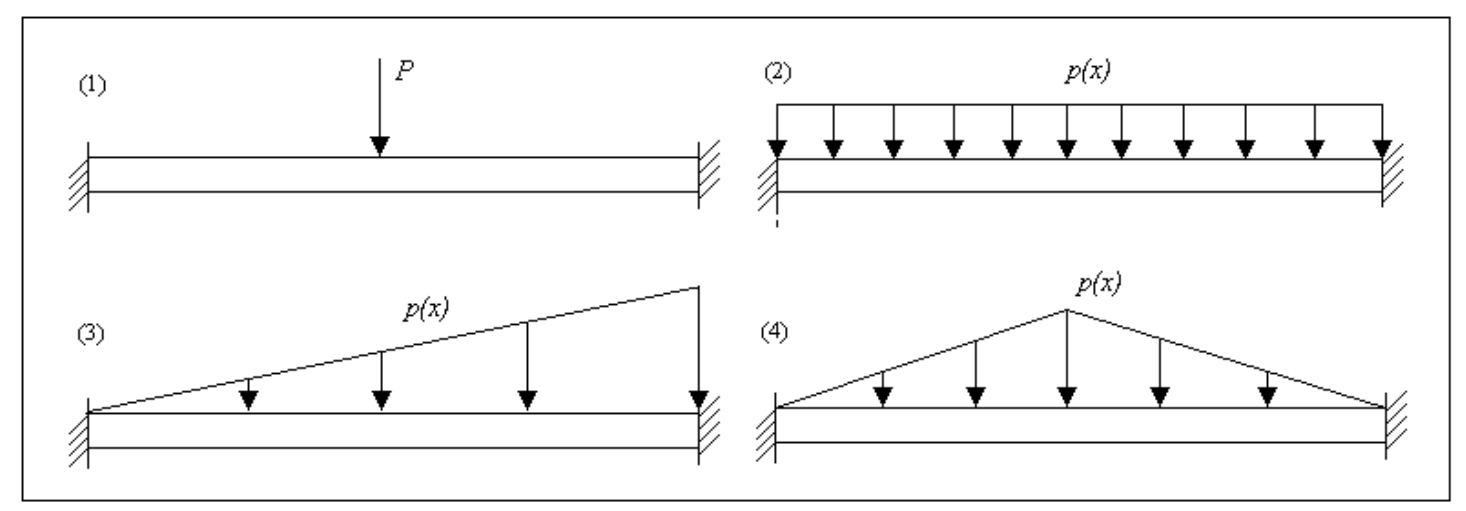

Figure 4. Different loading conditions applied on Beam-1: 1) Concentrated load, 2) Uniformly distributed load (UDL), 3) Triangular load and 4) Hat load.

\section{EXPERIMENT}

To validate the present method an experiment is carried out wherein a crossbeam structure is subjected to a concentrated load. The experimental setup comprises the following main components: channel frame, crossbeam structure, loading device and measurement instrument. Two slender beams are bolted firmly to the frame to make the crossbeam structure and the loading device is bolted to the frame and positioned over the crossbeam. This loading device consists of a loading platform fixed rigidly to a vertical loading rod with a pointed tip and a travelling arrangement with provisions for transverse and rotational adjustments for varying the point of application of the load. The travelling arrangement is a guideway (lubricated with oil) through which the loading rod can slide smoothly. The loading device is adjusted so that the tip of the loading rod just touches the upper beam under no loading condition and a dial gauge (Maker: Baker Mercer, Type: C02, Least Count: $0.01 \mathrm{~mm}$ ) is set under the structure at the desired position. Figures 5 and 6 show a photograph and a schematic diagram of the experimental setup. The load of dead weights placed on the loading platform, is transferred through the pointed tip of the rod onto the crossbeam structure and the resulting deflection is obtained from the reading of the dial gauge. 


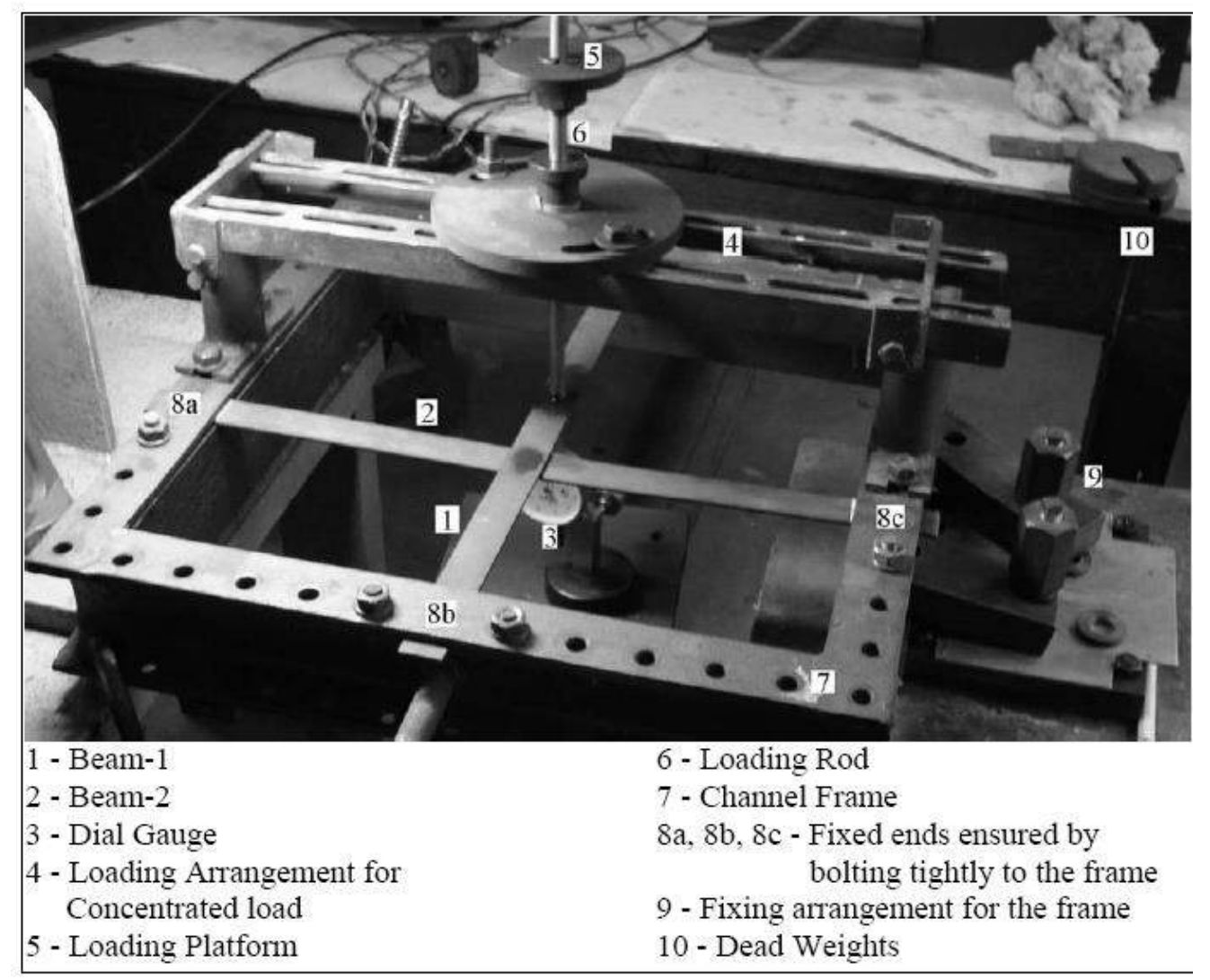

Figure 5. Photograph of the experimental set up.

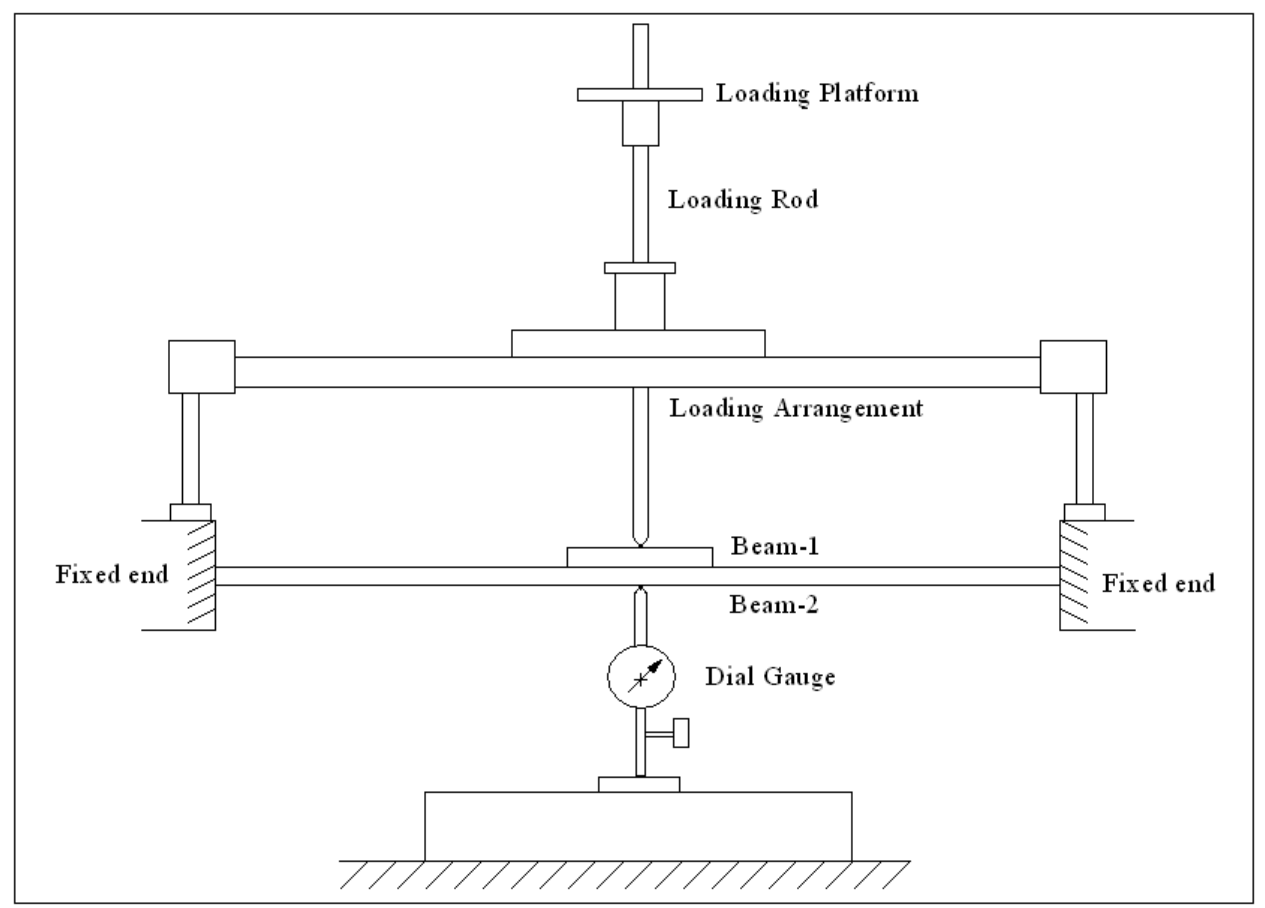

Figure 6. Schematic diagram of the experimental setup. 


\section{RESULTS AND DISCUSSION}

The effects of variations in beam thicknesses, loading pattern and position of Beam- 2 on the static behaviour of the system have been studied. The results are presented through plots of reaction force and displacement of the interaction point versus load. It is to be noted that in case of concentrated and uniformly distributed loads, the total load is considered, whereas for triangular and hat loads the maximum load is considered for the plots. In addition, the deflected shapes of the crossbeam structure at static equilibrium conditions, under a particular load, are provided.

The start functions for $w_{1}, u_{1}$ and $w_{2}, u_{2}$ are selected to satisfy the flexural and inplane boundary conditions of the beams. The start functions for the definition of beam deflection $\left(w_{1}, w_{2}\right)$ come from the flexural boundary conditions, i.e., both ends clamped. The start functions for the stretching of the beam $\left(u_{1}, u_{2}\right)$ come from the membrane boundary conditions and are assumed zero at the boundaries. Both sets of start functions are shown in Table 1. These selected start functions are used to generate the higher order functions with the help of the Gram-Schmidt orthogonalisation principle.

Table 1. Start functions for $w_{1}, u_{1}$ and $w_{2}, u_{2}$.

\begin{tabular}{ccc}
\hline \multirow{2}{*}{ Beam-1 } & $\phi_{11}(\xi)$ & $\{\xi(1-\xi)\}^{2}$ \\
\cline { 2 - 3 } Beam-2 & $\alpha_{11}(\xi)$ & $\xi(1-\xi)$ \\
\hline \multirow{2}{*}{ Bear } & $\phi_{21}(\eta)$ & $\{\eta(1-\eta)\}^{2}$ \\
\hline
\end{tabular}

The number of functions ( $n w$ and $n u$ ) to be used is determined from a convergence study, where a crossbeam structure (Dimensions: $L_{1}=1 \mathrm{~m}, b_{1}=0.02 \mathrm{~m}, t_{1}=0.005 \mathrm{~m}$ and $L_{2}=1 \mathrm{~m}, b_{2}=0.02 \mathrm{~m}, t_{2}=0.005 \mathrm{~m}$, Material property: $E_{1}=E_{2}=210 \mathrm{GPa}, \rho_{1}=\rho_{2}=7850 \mathrm{~kg} / \mathrm{m}^{3}$, Support Locations: $x_{r}=0.5 L_{1}$ and $y_{r}=0.5 L_{2}$ ) under uniformly distributed loading is analysed for variations in number of functions. The results, provided in Figure 7, show plots of displacement vs. load for variations in number of functions and thus, a value of $n w=n u=8$ is chosen for further studies. Also, the error limit $\left(\varepsilon_{1}\right)$ for convergence of individual beams is taken as 0.01 and the error limit $\left(\varepsilon_{2}\right)$ for convergence of the outer loop, i.e., for $\left(\delta_{b 1}-\delta_{b 2}\right) / t_{\min }$ is taken as 0.001 . 


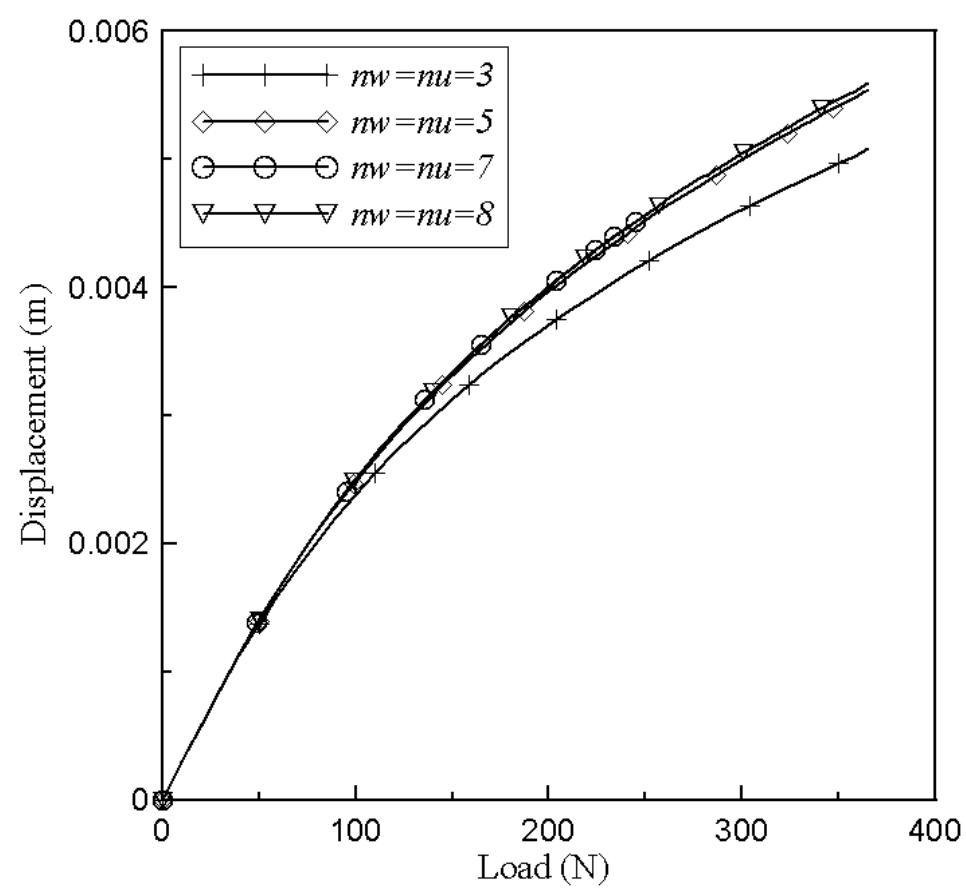

Figure 7. Plots for Displacement vs. Load for variation in number of functions Loading type: UDL, Beam- 1 dimensions: $L_{1} \times b_{1} \times t_{1}=1 \times 0.02 \times 0.005$ (in m), Beam-2 dimensions: $L_{2} \times b_{2} \times t_{2}=1 \times 0.02 \times 0.005$ (in m), Support locations: $x_{r}=0.5 L_{1}$ and

\section{Validation Study}

$$
y_{r}=0.5 L_{2} \text {. }
$$

The crossbeam structure subjected to concentrated loading in the performed experiment comprises two slender beams with the following dimensions: Beam-1: $L_{1}=400 \mathrm{~mm}, b_{1}$ $=24.7 \mathrm{~mm}, t_{1}=3 \mathrm{~mm}$ and Beam-2: $L_{2}=400 \mathrm{~mm}, b_{2}=24.7 \mathrm{~mm}, t_{2}=3 \mathrm{~mm}$. The beam material is mild steel and the material properties are assumed as $E_{1}=E_{2}=210 \mathrm{GPa}$, $\rho_{1}=\rho_{2}=7850 \mathrm{~kg} / \mathrm{m}^{3}$. Experiments are carried out for two positions of Beam-2, which are $x_{r}=0.5 L_{1}$ and $x_{r}=0.335 L_{1}$. The concentrated load is applied on Beam-1 over the contact point between the beams i.e,. $x_{p}=0.5 L_{1}$ and $x_{p}=0.335 L_{1}$ for the respective cases and the dial gauge is placed directly below the point of application of the load, touching the lower surface of Beam-2. Figure 8(a) and (b) compares the experimental data with the results generated through the present method for the two cases mentioned above. Figure 9 shows the displacement-load plots for experimental data and the present method for a case where Beam- 2 is positioned at $x_{r}=0.335 L_{1}$ but the load is applied at a different location $\left(x_{p}=0.5 L_{1}\right)$. In this case, the dial gauge is set below the point of load application but in contact with Beam-1. These figures show that in all the experimental results, the nature of the displacement vs. load curves is similar to those predicted by the present method but the actual displacement values are higher. This variation may be because the stretching boundary conditions are not satisfied. By bolting the ends of the beams, zero transverse displacement is ensured but the same cannot be said about the axial displacement conditions. Additionally, the difference between the results of the experimental and present method increases with an increase in load, which also indicates that at higher loads, the stretching forces come into effect. 


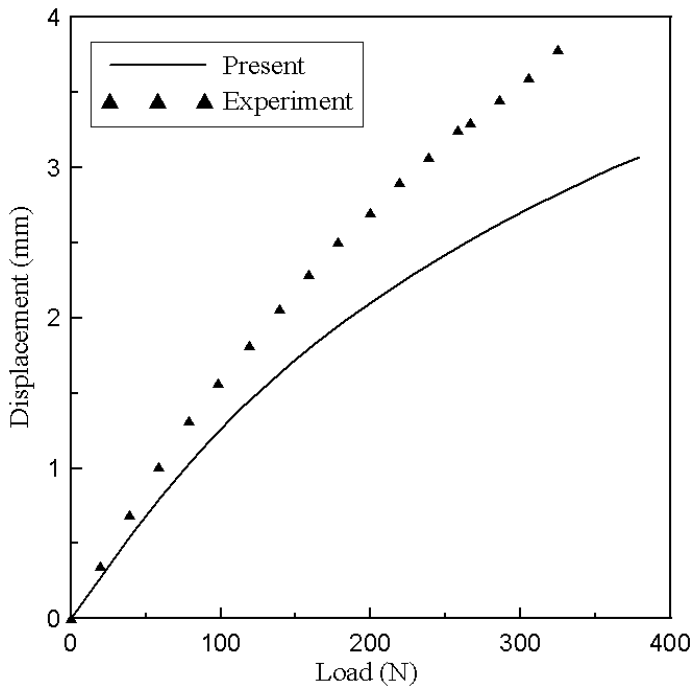

(a)

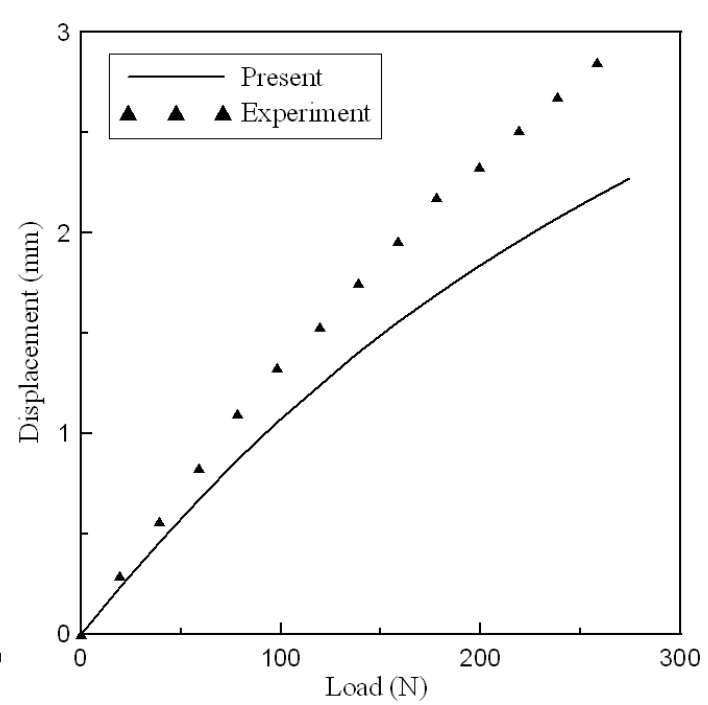

(b)

Figure 8. Comparison of experimental results with the present method - Plots for Displacement vs. Load for (a) $x_{r}=0.5 L_{1}, x_{p}=0.5 L_{1}$ and (b) $x_{r}=0.335 L_{1}, x_{p}=0.335 L_{1}$ with Loading type: Concentrated, Beam- 1 dimensions: $L_{1} \times b_{1} \times t_{1}=400 \times 24.7 \times 3$ (in $\mathrm{mm}$ ), Beam-2 dimensions: $L_{2} \times b_{2} \times t_{2}=400 \times 24.7 \times 3$ (in mm).

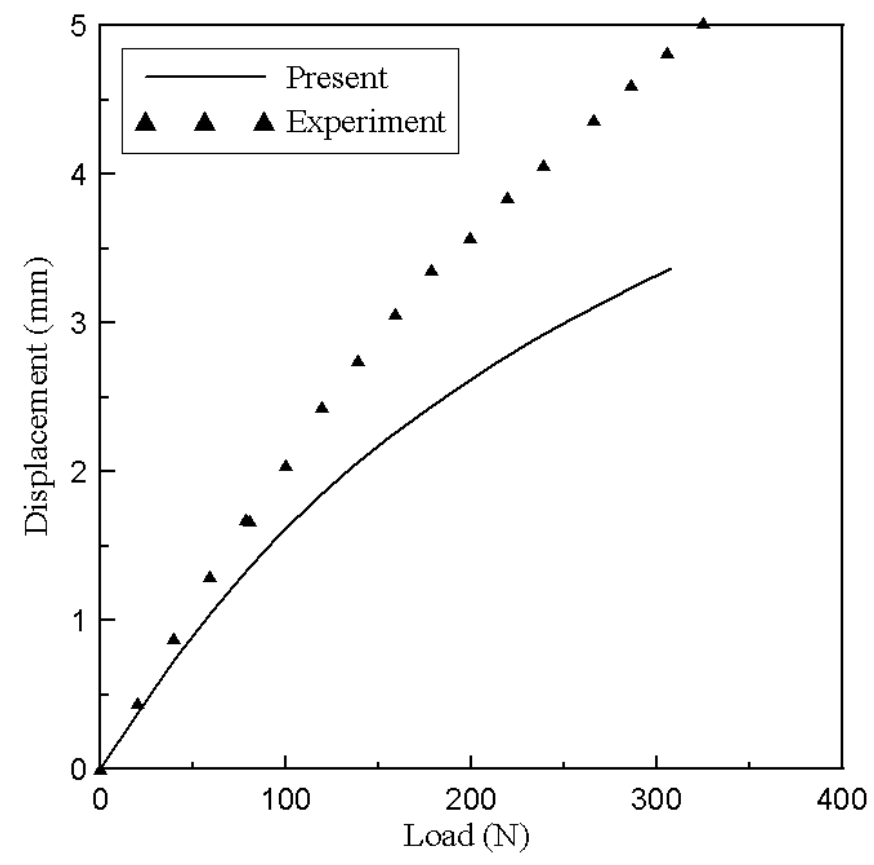

Figure 9. Comparison of experimental results with the present method - Plots for Displacement vs. Load for $x_{r}=0.335 L_{1}, x_{p}=0.5 L_{1}$ with Loading type: Concentrated,

Beam-1 dimensions: $L_{1} \times b_{1} \times t_{1}=400 \times 24.7 \times 3$ (in mm), Beam-2 dimensions:

$$
L_{2} \times b_{2} \times t_{2}=400 \times 24.7 \times 3(\text { in } \mathrm{mm}) \text {. }
$$

2-D Analysis: The beams are individually modelled as 2-D elements and separately analysed with the reaction force $(R)$ being used as an input. Comparing the displacement fields produced with those generated by the present method shows 
excellent agreement. Figure 10 shows the load-displacement plots corresponding to the contact point of the beams.

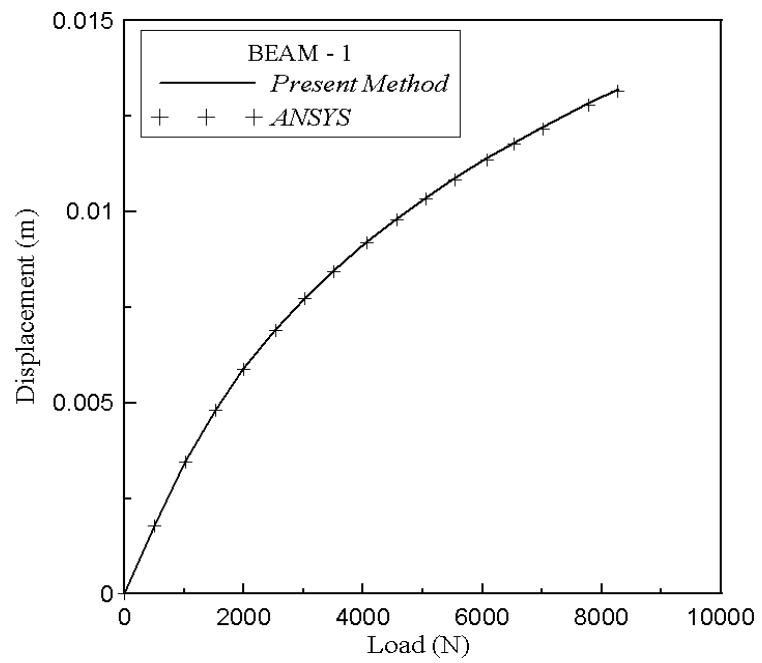

(a)

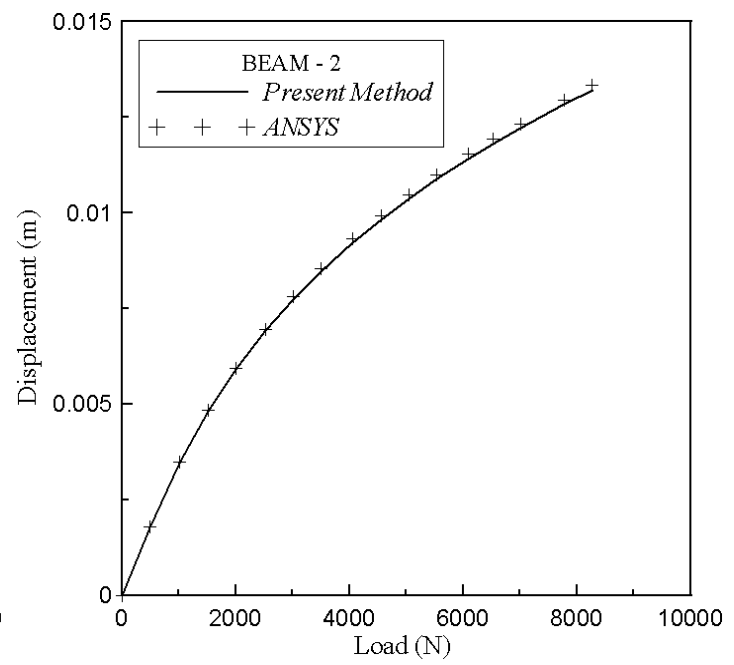

(b)

Figure 10. Validation plots with ANSYS 2-D analysis for Displacement vs. Load for (a) Beam-1 and (b) Beam-2 - Loading type: UDL, Beam-1 dimensions:

$L_{1} \times b_{1} \times t_{1}=1 \times 0.02 \times 0.01$ (in m), Beam-2 dimensions: $L_{2} \times b_{2} \times t_{2}=1 \times 0.02 \times 0.01$ (in m), Support locations: $x_{r}=0.5 L_{1}$ and $y_{r}=0.5 L_{2}$.

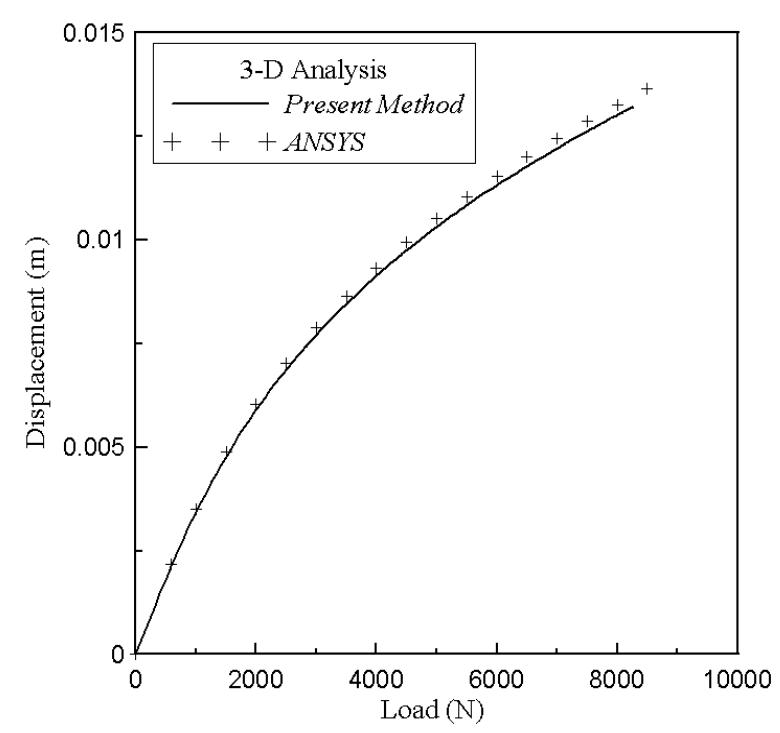

Figure 11. Validation plot with ANSYS 3-D analysis for Displacement (at the contact point between the beams) vs. Load with Loading type: UDL, Beam-1 dimensions: $L_{1} \times b_{1} \times t_{1}=1 \times 0.02 \times 0.01$ (in m), Beam-2 dimensions: $L_{2} \times b_{2} \times t_{2}=1 \times 0.02 \times 0.01$ (in m), Support locations: $x_{r}=0.5 L_{1}$ and $y_{r}=0.5 L_{2}$.

3-D Analysis: A 3-D model of the crossbeam with the previously mentioned geometry, generated in solid modelling software, is exported to the ANSYS Workbench (Version 11.0) and the analysis performed under uniformly distributed loading and fixed end conditions. The simulated displacement field has good agreement with the displacement field obtained by the present method. Figure 11 shows the displacement- 
load plot at the interaction point between the two beams for ANSYS and the present method. The matching of the results in both the cases of the 2-D and 3-D analysis are found to be excellent and establishes the present method within the limitations of the mathematical boundary conditions. Effects of different system parameters are studied and displacement-load plots are furnished in each case along with the visualisation of the deflected shape.

\section{Variation in Beam-1 Thickness}

To investigate changes in the static response of the system due to variations in the thickness of Beam-1, other dimensions, loading pattern and end conditions are kept constant. The reaction-load and displacement-load plots of the system are shown in dimensional form, in Figure 12(a) and (b), respectively. As mentioned earlier, the displacement at the point of contact between the two beams is considered as the system displacement. Figure 12(a) shows that for a thicker Beam-1, reaction force is low and it increases with a decrease in the thickness of Beam-1. This is because the thicker upper beam takes up a greater portion of the external load and thus, generates lower reaction force. Figure 12(b) shows that the displacement-load curves become steeper for lower Beam-1 thickness, i.e., the stiffness of the system reduces. Figure 13 shows the deflected shapes for each of the cases considered in Figure 12(a) and (b), corresponding to the maximum load (denoted by points $\mathrm{A}, \mathrm{B}, \mathrm{C}$ and $\mathrm{D}$ in the displacement-load plots of Figure 12(b)).

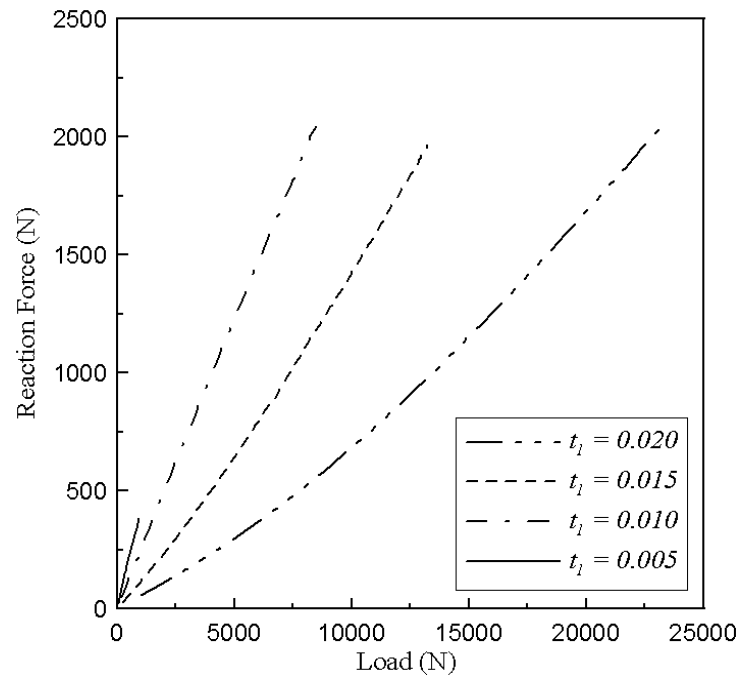

(a)

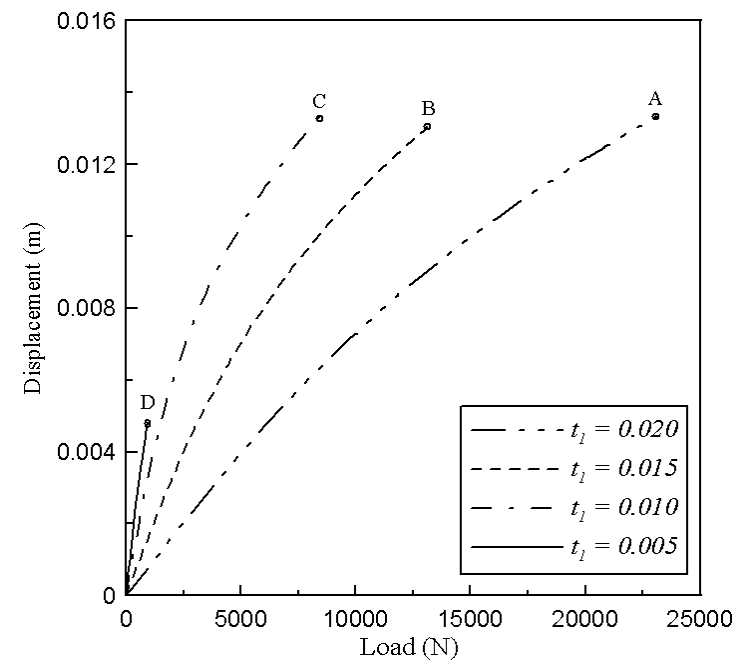

(b)

Figure 12. Plots for (a) Reaction force vs. Load and (b) Displacement vs. Load for variation of thickness of Beam-1 $\left(t_{1}\right)$ - Loading type: UDL, Beam-1 dimensions:

$L_{1} \times b_{1} \times t_{1}=1 \times 0.02 \times t_{1}$ (in m), Beam-2 dimensions: $L_{2} \times b_{2} \times t_{2}=1 \times 0.02 \times 0.01$ (in m), Support locations: $x_{r}=0.5 L_{1}$ and $y_{r}=0.5 L_{2}$. 


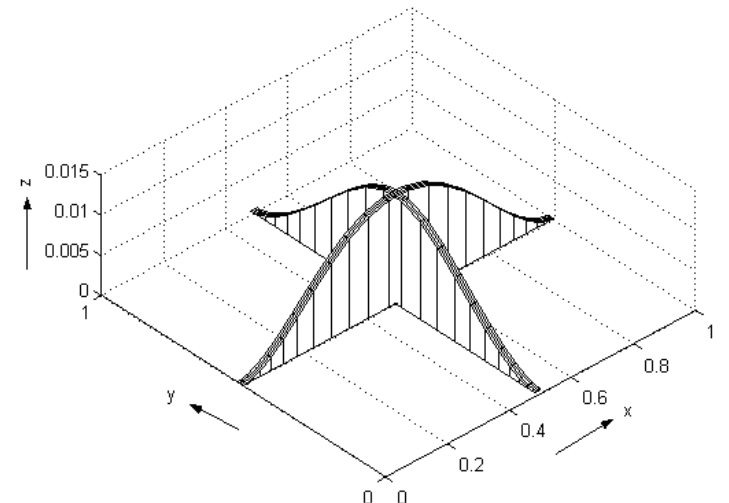

(a)

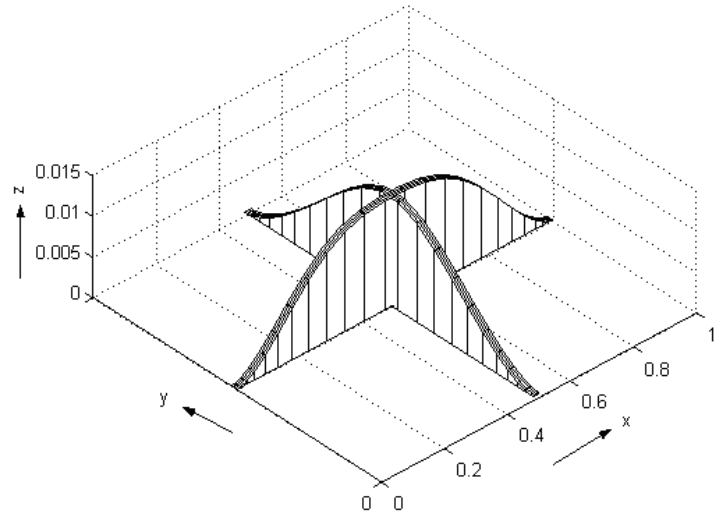

(c)

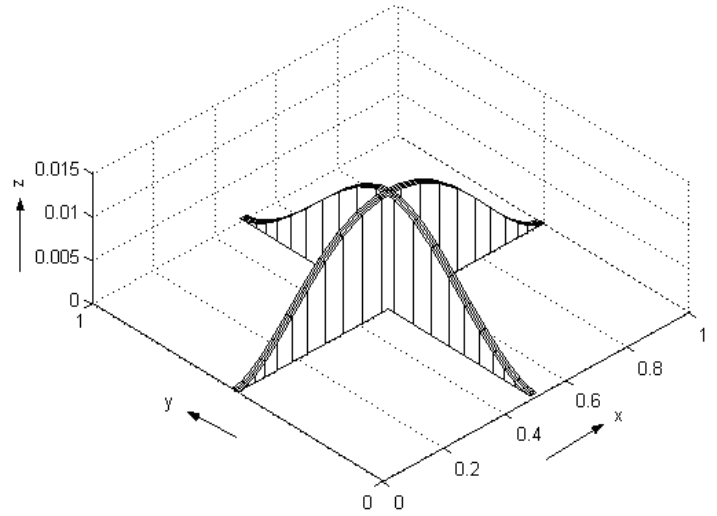

(b)

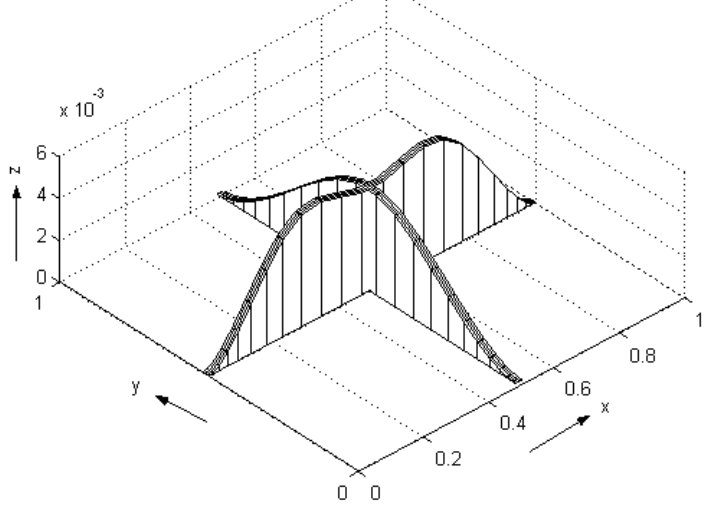

(d)

Figure 13. Deflected shapes of the structure under loading for variation of Beam-1 thickness - (a) $t_{1}=0.020$, (b) $t_{1}=0.015$, (c) $t_{1}=0.010$, (d) $t_{1}=0.005$, taken at points A, B, C and D in the displacement-load plots of Figure 12(b).

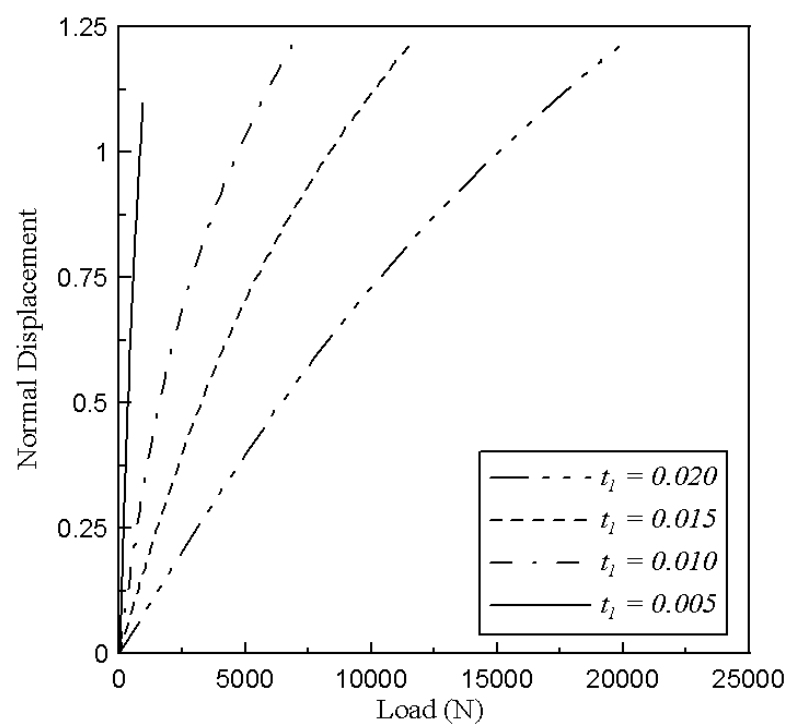

Figure 14. Plots for normalised maximum displacement of the system vs. load for variation of thickness of Beam-1 $\left(t_{1}\right)$ - Loading type: UDL, Beam-1 dimensions: $L_{1} \times b_{1} \times t_{1}=1 \times 0.02 \times t_{1}$ (in m), Beam-2 dimensions: $L_{2} \times b_{2} \times t_{2}=1 \times 0.02 \times 0.01$ (in m),

Support locations: $x_{r}=0.5 L_{1}$ and $y_{r}=0.5 L_{2}$. 
It may appear from Figure 12(b) that for $t_{1}=0.005 \mathrm{~m}$, the deformation of the system is low compared with the other cases. However, it is to be noted that this figure depicts the displacement of the contact point of the beams in dimensional form, whereas the maximum deflection of the system is at a different location along Beam-1 (clearly shown in Figure 13(d)). If the maximum displacement of the system (may be on Beam1 or Beam-2) normalised by the corresponding beam thickness is considered, it is seen that the degree of nonlinearity achieved is more or less same. This is elaborated in Figure 14, where the plots for normalised maximum displacement of the system versus load are shown for variations of thickness of Beam-1 $\left(t_{1}\right)$.

\section{Variation in Beam-2 Thickness}

Investigations of the effects of variation in the thickness of Beam-2 are carried out following the same procedure and the reaction-load and displacement-load plots of the system in dimensional form, are shown in Figure 15(a) and (b). Figure 15(a) clearly shows that for a thicker Beam-2 a large reaction force is generated and lower reaction forces occur as the thickness decreases. This behaviour may seem contrary to the previous case but in actual effect, these two cases are identical. In relative terms, a decrease in Beam-2 thickness amounts to an increase in Beam-1 thickness. Therefore, it is observed that for a particular loading and boundary conditions, the reaction force generated depends on the relative geometry of the two beams. Generally, a thicker Beam-1 (compared with Beam-2) means a lower reaction force and a thinner Beam-1 generates a higher reaction force. Figure 15(b) shows that for a greater thickness of Beam-2, the displacement of the contact point is lower for the same load, which means that the stiffening effect of Beam-2 increases with an increase in its thickness. The deflected shapes of the structure under loading, corresponding to the points A, B, C and D (representing maximum loads) in Figure 15(b), are shown in Figure 16.

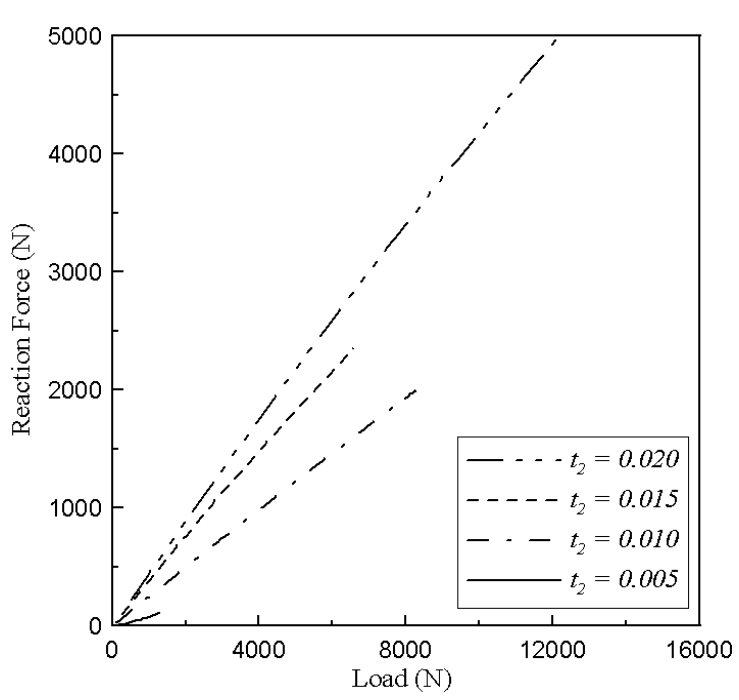

(a)

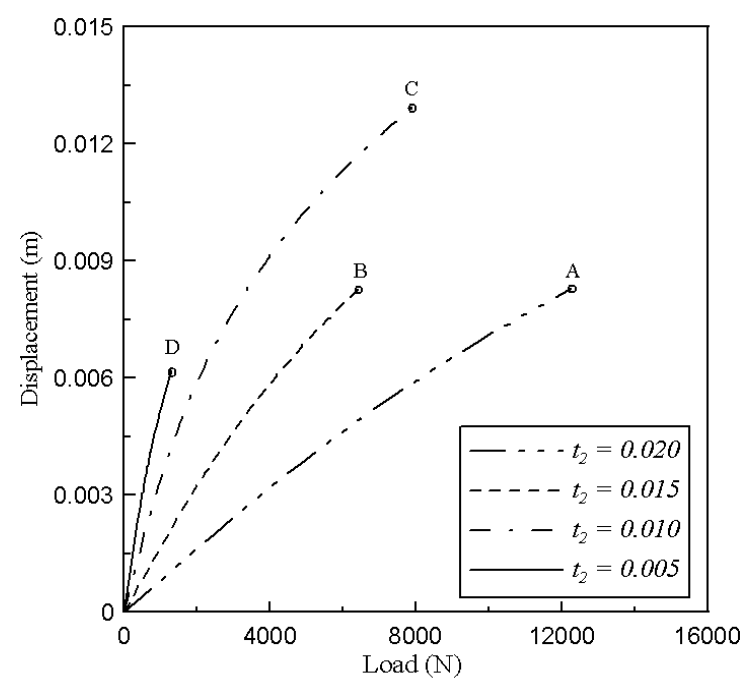

(b)

Figure 15. Plots for (a) Reaction force vs. Load and (b) Displacement vs. Load for variation of thickness of Beam-2 $\left(t_{2}\right)$ - Loading type: UDL, Beam-1 dimensions:

$L_{1} \times b_{1} \times t_{1}=1 \times 0.02 \times 0.01$ (in m), Beam-2 dimensions: $L_{2} \times b_{2} \times t_{2}=1 \times 0.02 \times t_{2}$ (in m), Support locations: $x_{r}=0.5 L_{1}$ and $y_{r}=0.5 L_{2}$. 


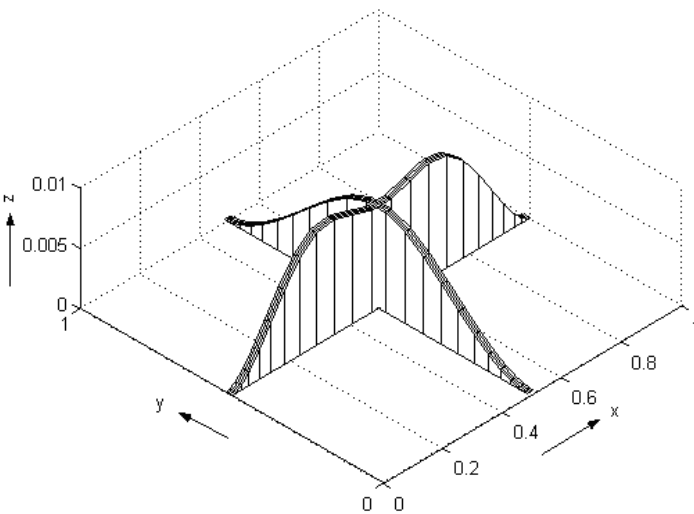

(a)

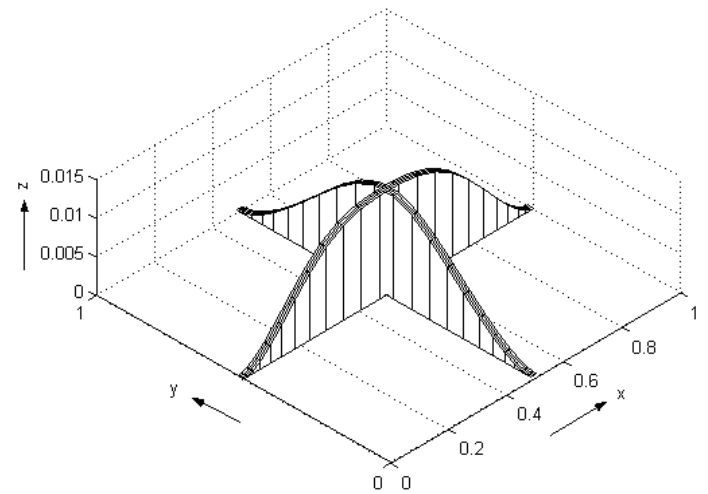

(c)

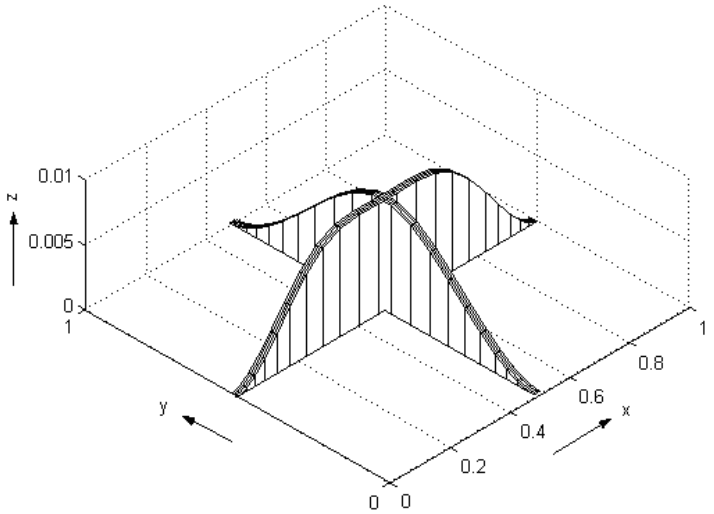

(b)

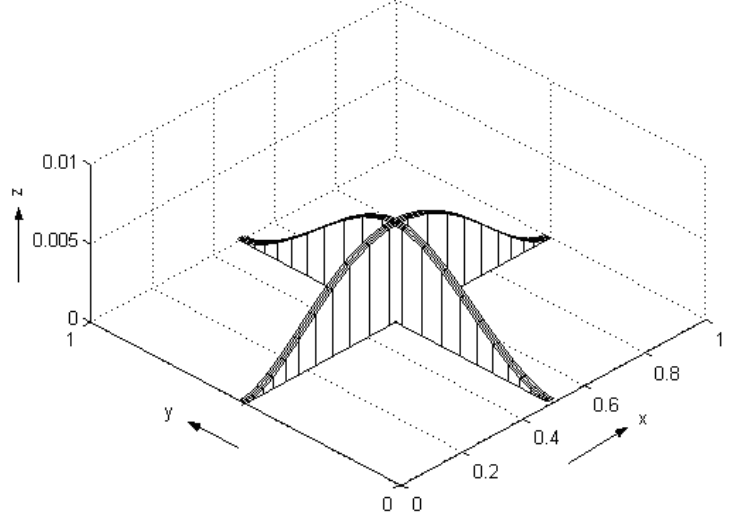

(d)

Figure 16. Deflected shapes of the structure under loading for variation of Beam-2 thickness - (a) $t_{2}=0.020$, (b) $t_{2}=0.015$, (c) $t_{2}=0.010$, (d) $t_{2}=0.005$, taken at points A, B, C and D in the displacement-load plots of Figure 15(b).

\section{Variation in Beam-2 Position}

The system behaviour for different positions of Beam-2 along the span of Beam-1 is analysed. For this case, all the boundary conditions are taken as clamped and the loading type is UDL. The dimensions of the two beams are kept constant at $L_{1} \times b_{1} \times t_{1}=1 \times 0.02 \times 0.01$ and $L_{2} \times b_{2} \times t_{2}=1 \times 0.02 \times 0.01$ (all dimension are in $m$ ). Positions of Beam-2 are varied along one side of the mid-span of Beam-1, as the geometry is symmetric. Figure 17(a) and (b) shows the reaction-load and displacementload plots of the system in dimensional form, respectively. From Figure 17(a) it can be said that higher reaction force is generated when Beam-2 is positioned at the mid-span of Beam-1 and as it shifts towards one of the ends, the reaction force becomes lower. Figure 17(b) shows that for a particular load, the displacement of the contact point is higher near the mid-span position of Beam-2 than the positions towards the ends. However, the contact point displacement might not be the maximum displacement along Beam-1. This is shown through the deflected shapes illustrated in Figure 18, for different positions of Beam-2. In addition, the plots of maximum displacement-load are provided along with the displacement-load plot of the contact point. From these figures, it is evident that as Beam-2 shifts towards the ends, the contact point displacement decreases but maximum displacement of the system increases. Therefore, the optimum stiffening effect may be obtained from a centrally placed supporting beam. 


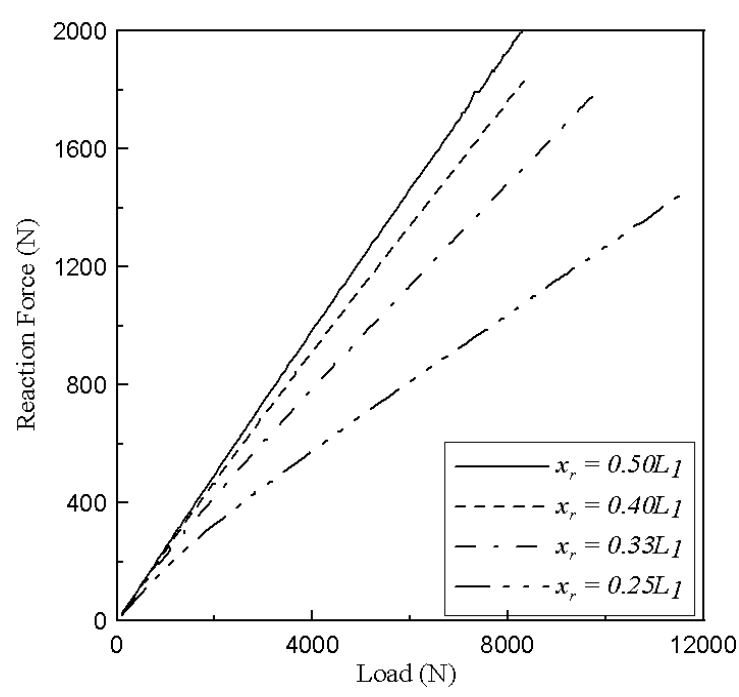

(a)

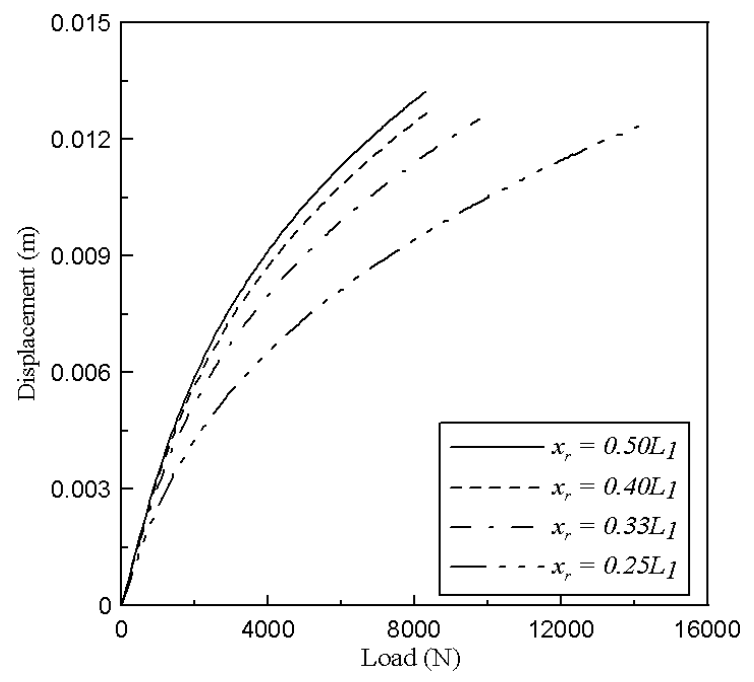

(b)

Figure 17: Plots for (a) Reaction force vs. Load and (b) Displacement vs. Load for variation of Beam-2 position $\left(x_{r}\right)$ - Loading type: UDL, Beam-1 dimensions:

$L_{1} \times b_{1} \times t_{1}=1 \times 0.02 \times 0.01$ (in m), Beam-2 dimensions: $L_{2} \times b_{2} \times t_{2}=1 \times 0.02 \times 0.01$ (in $\mathrm{m})$, Support locations: $y_{r}=0.5 L_{2}$.

\section{Variation in Loading Pattern}

The effect of different loading patterns on the crossbeam structure is investigated for two different geometries of Beam-1 and Beam-2. The position of Beam-2 is kept fixed at the mid-span of Beam-1, i.e., $x_{r}=0.50$. For the two different beam geometries, Figures 19(a) and 20(a) plot the reaction force vs. load for various types of loading. It is evident from these figures that in both cases the concentrated load generates the highest reaction force, whereas for triangular loading the reaction force is lowest. The plots of displacement vs. load for the two geometries are presented in Figures 19(b) and 20(b). The deflected shapes of the structure for different types of loading, corresponding to the second geometry (Figure 20), are given in Figure 21. Additionally, in this case it is observed that the point of interaction of the two beams may not be the point of maximum displacement of the structure. This is in fact dependent both on the relative geometry of the two beams and the nature of loading applied to Beam-1. For example, a concentrated load applied over the point of interaction of the two beams, irrespective of the geometry, produces the maximum deflection of the system at the point of interaction. However, for symmetrically distributed loading (UDL and Hat load), the relative geometry of the beams becomes important in determining the point of maximum deflection. For asymmetrically distributed loading (Triangular load) or a concentrated load, applied at locations other than the interaction point, the maximum deflection is likely to occur away from the contact point of the beams. 

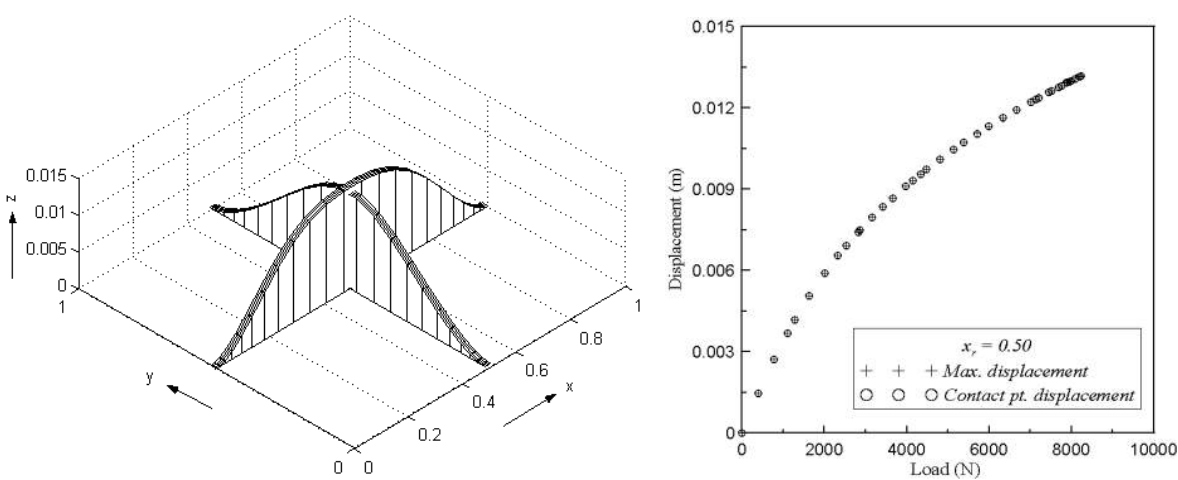

(a)
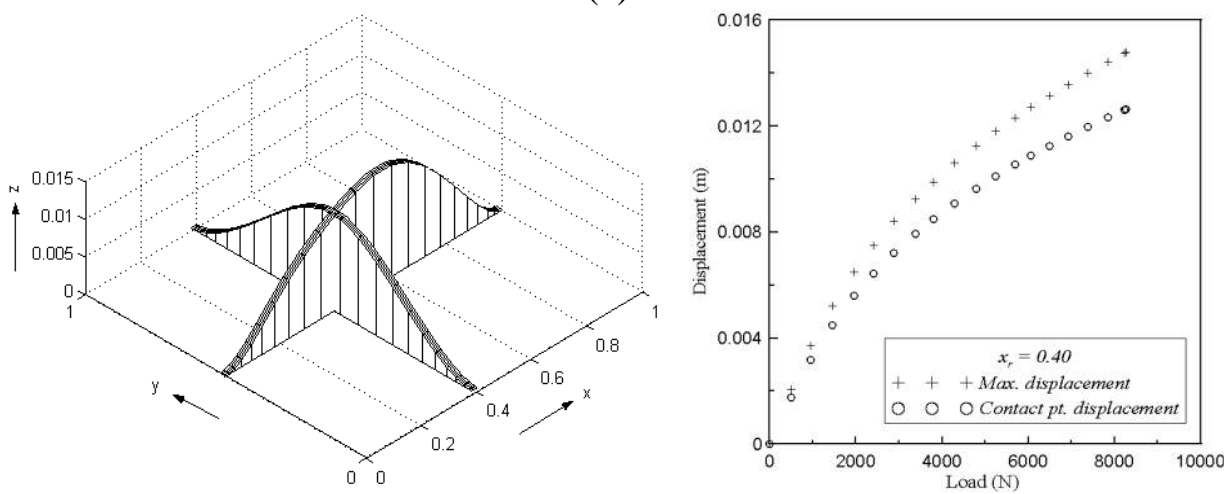

(b)
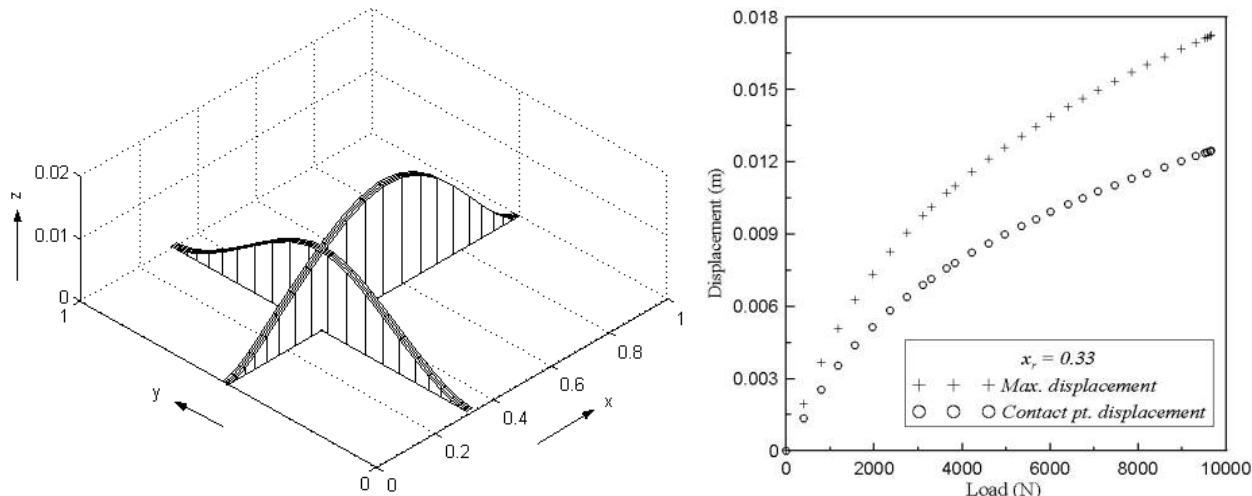

(c)
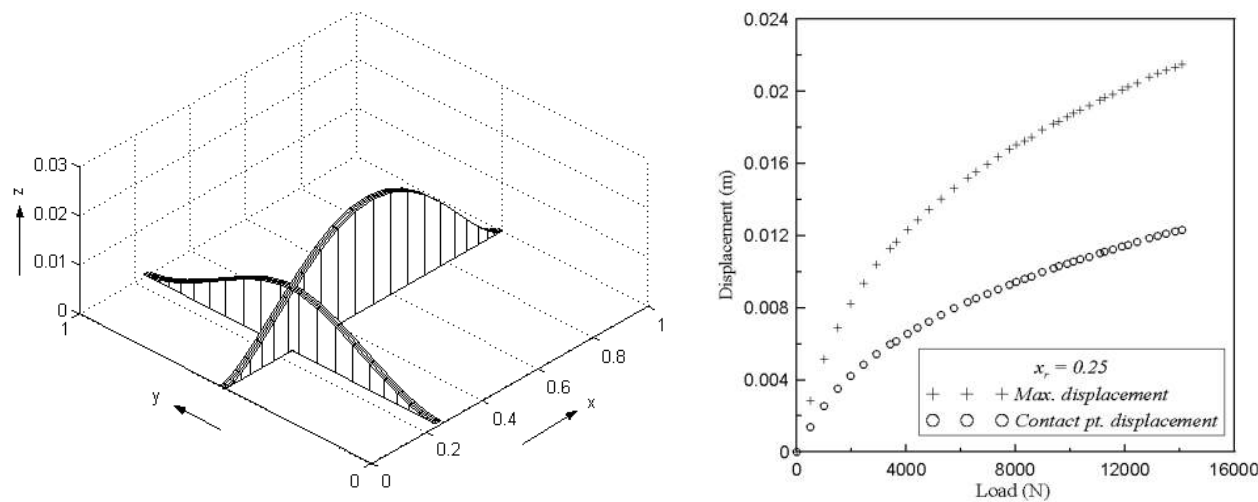

(d)

Figure 18. Deflected shapes of the structure under loading for variation of Beam-2 position along with maximum displacement and contact point displacement comparisons - (a) $x_{r}=0.50 L_{1}$, (b) $x_{r}=0.40 L_{1}$, (c) $x_{r}=0.33 L_{1}$, (d) $x_{r}=0.25 L_{1}$. 


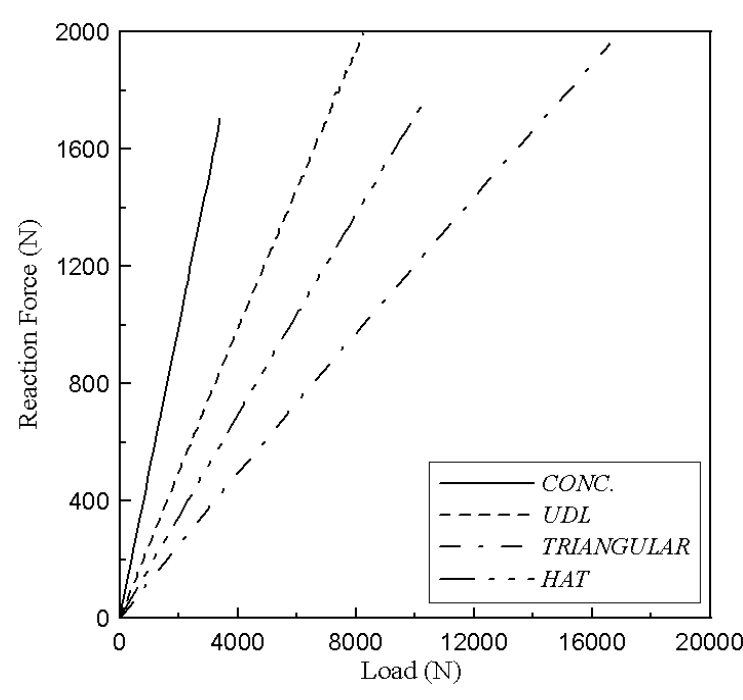

(a)

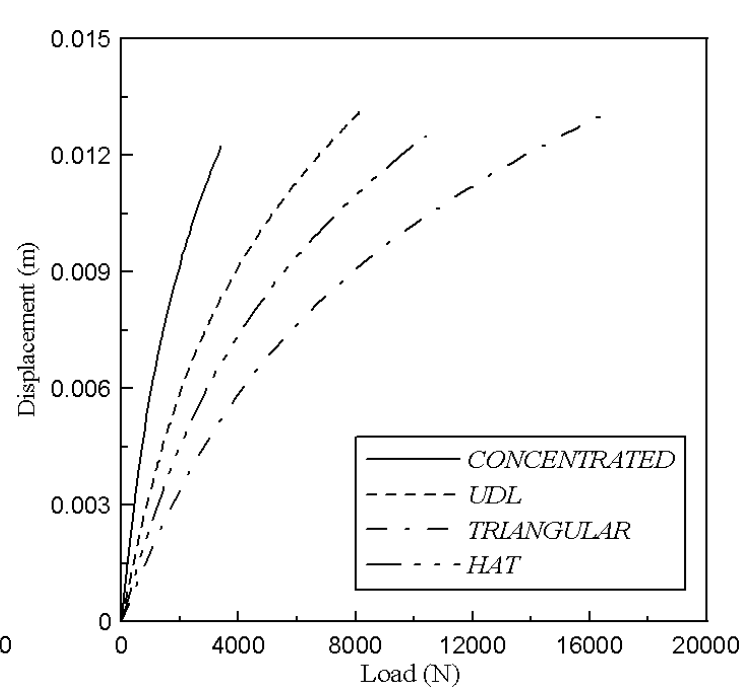

(b)

Figure 19. Plots for (a) Reaction force vs. Load and (b) Displacement vs. Load for different loading pattern - Beam-1 dimensions: $L_{1} \times b_{1} \times t_{1}=1 \times 0.02 \times 0.01$ (in m), Beam2 dimensions: $L_{2} \times b_{2} \times t_{2}=1 \times 0.02 \times 0.01$ (in m), Support locations: $x_{r}=0.5 L_{1}$ and

$$
y_{r}=0.5 L_{2} \text {. }
$$

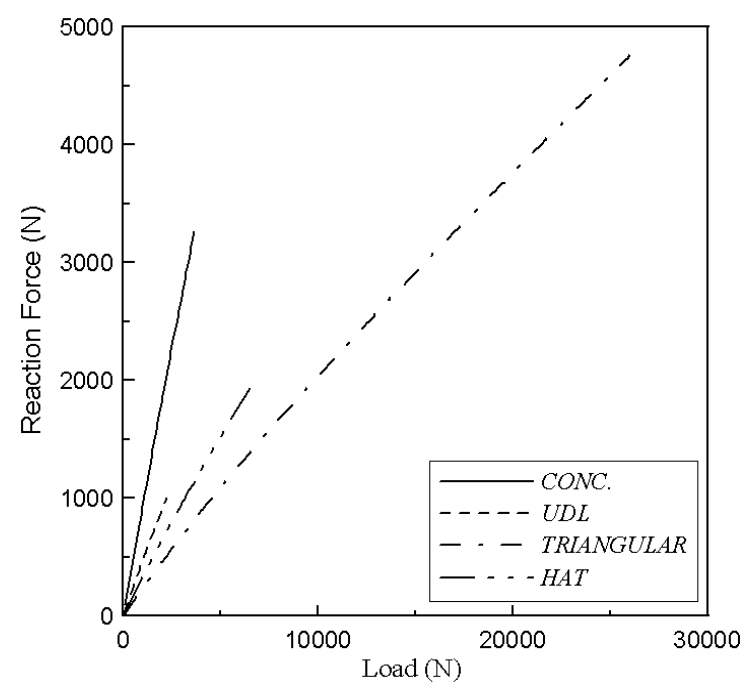

(a)

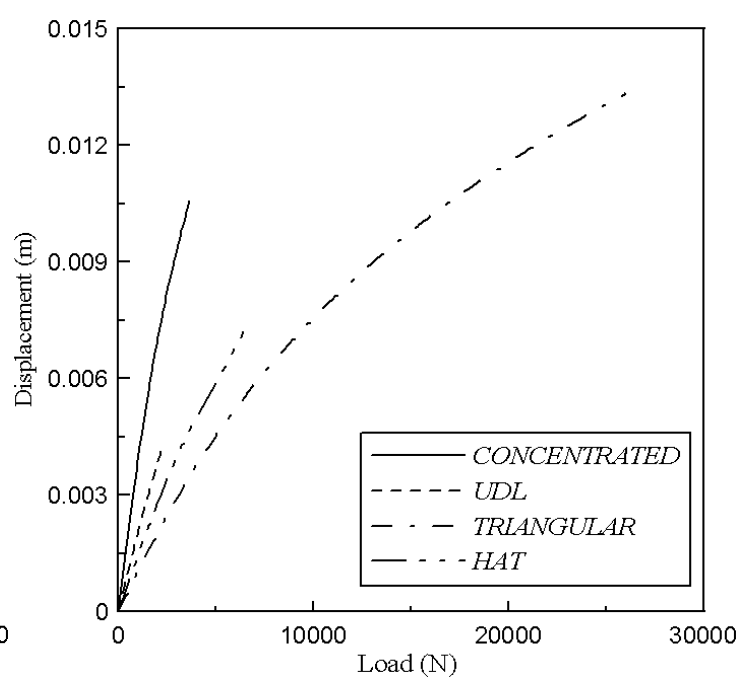

(b)

Figure 20. Plots for (a) Reaction force vs. Load and (b) Displacement vs. Load for different loading pattern - Beam-1 dimensions: $L_{1} \times b_{1} \times t_{1}=1 \times 0.02 \times 0.005$ (in m),

Beam-2 dimensions: $L_{2} \times b_{2} \times t_{2}=1 \times 0.02 \times 0.015$ (in m), Support locations: $x_{r}=0.5 L_{1}$ and $y_{r}=0.5 L_{2}$. 

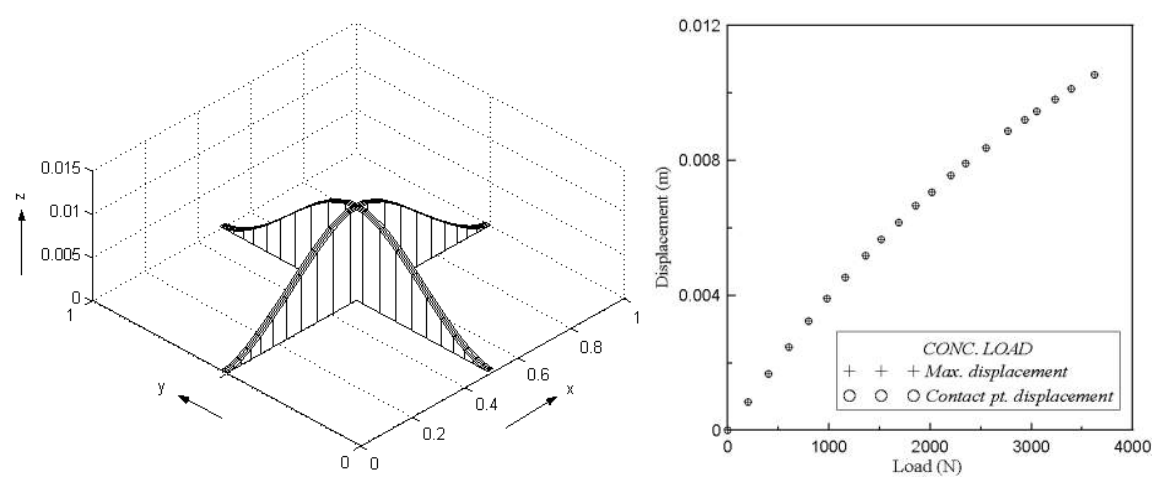

(a)
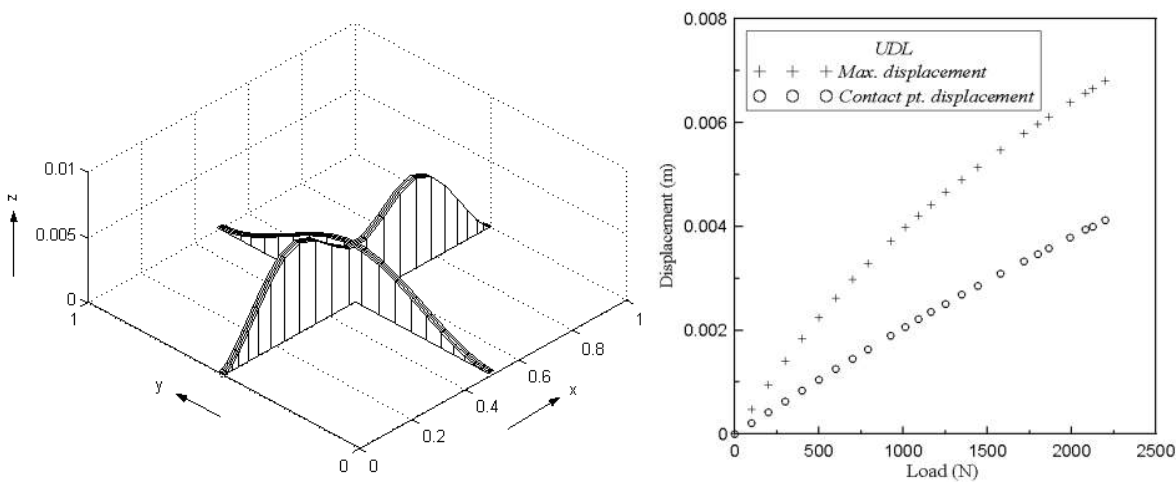

(b)
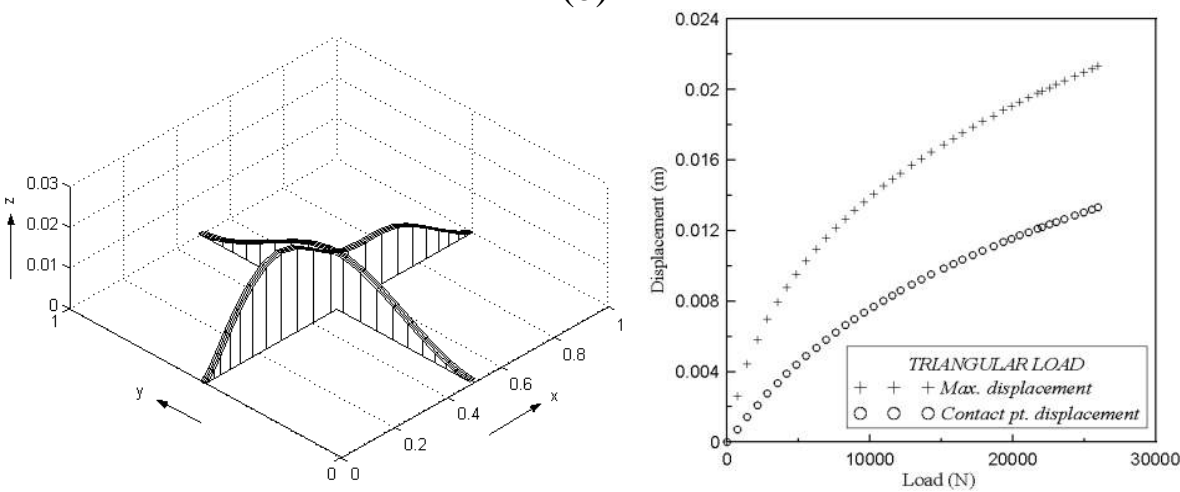

(c)
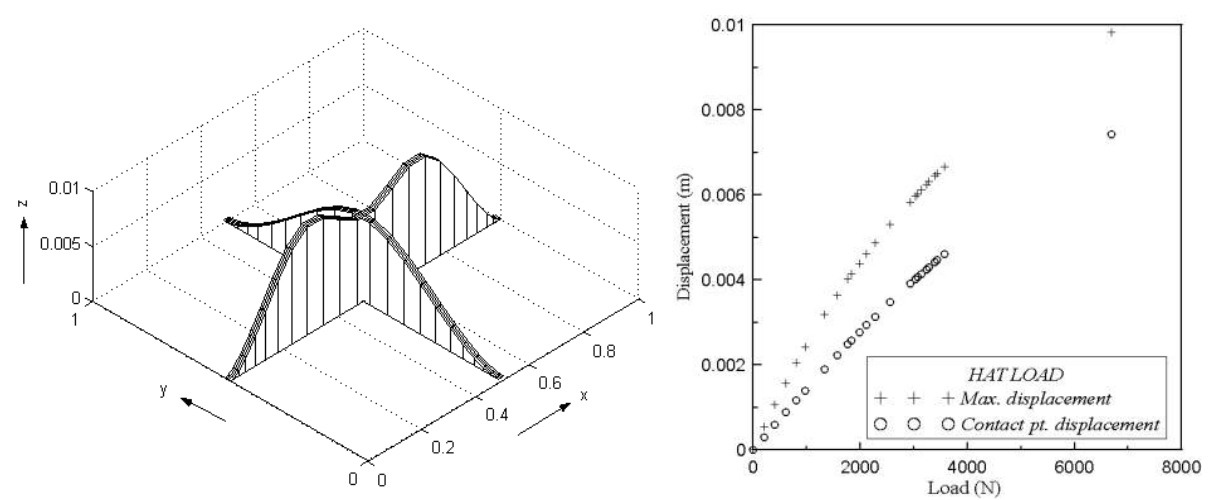

(d)

Figure 21. Deflected shapes of the structure under loading for various types of loading pattern along with maximum displacement and contact point displacement comparisons - (a) Concentrated load, (b) Uniformly distributed load (UDL), (c) Triangular load, (d) Hat load. 
Figure 22(a) shows the variation of maximum axial displacement of the two beams for a uniformly distributed loading and a given geometry of the structure. It is evident that the axial displacements for Beam-2 are considerably lower than that for Beam-1. Figure 22(b), which represents the distribution of the axial displacements along the beams for maximum load, highlights the low stretching of Beam-2 compared with Beam-1. This directly relates to the geometry of the beams and a thinner beam would have larger stretching in comparison with a thicker beam. The axial displacement fields for the two beams are symmetrical about their mid-point.

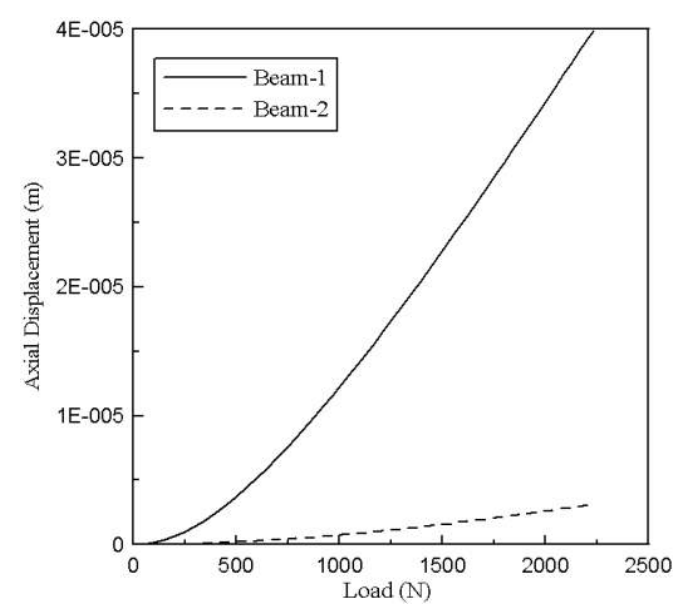

(a)

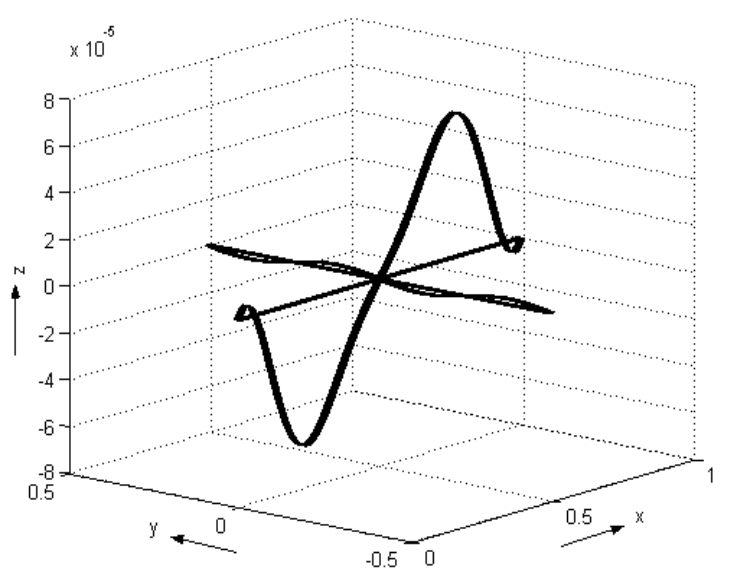

(b)

Figure 22. Plots for (a) Maximum axial displacement vs. Load and (b) Distribution of axial displacement along the beams for - Loading type: UDL, Beam-1 dimensions:

$$
L_{1} \times b_{1} \times t_{1}=1 \times 0.02 \times 0.005 \text { (in } \mathrm{m} \text { ), Beam-2 dimensions: }
$$

$L_{2} \times b_{2} \times t_{2}=1 \times 0.02 \times 0.015$ (in m), Support locations: $x_{r}=0.5 L_{1}$ and $y_{r}=0.5 L_{2}$.

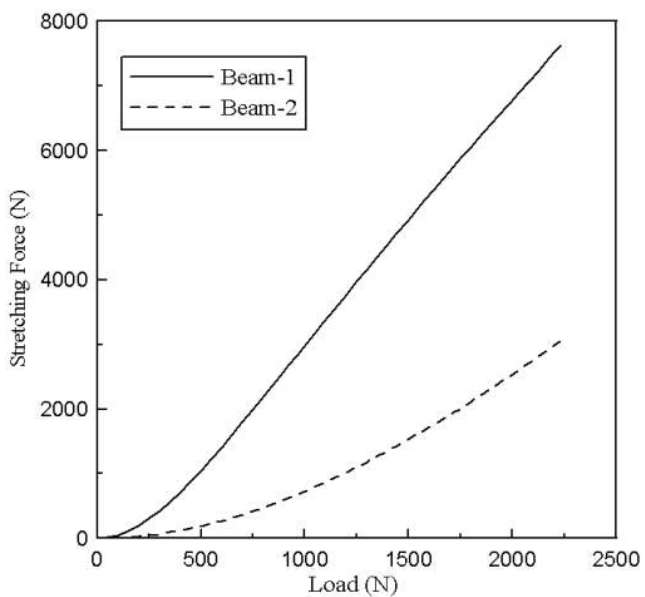

(a)

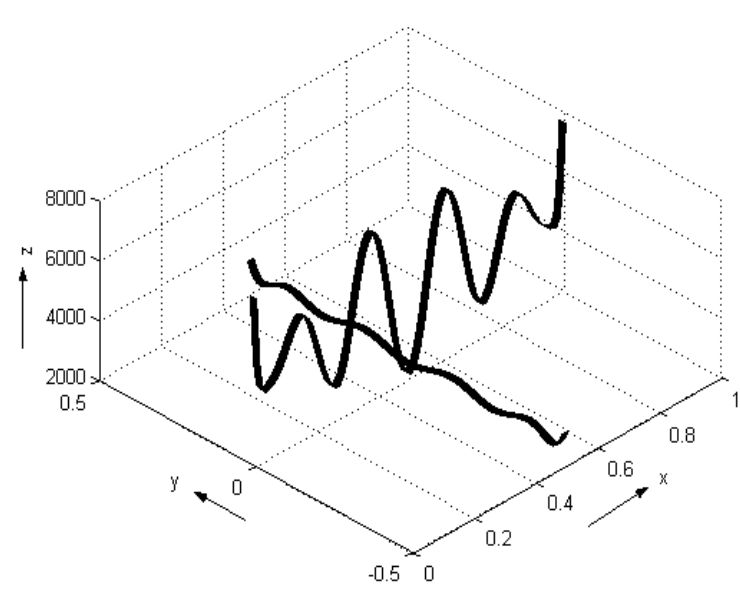

(b)

Figure 23. Plots for (a) Maximum stretching force vs. Load and (b) Distribution of stretching force along the beams for - Loading type: UDL, Beam-1 dimensions:

$$
L_{1} \times b_{1} \times t_{1}=1 \times 0.02 \times 0.005 \text { (in m), Beam-2 dimensions: }
$$

$L_{2} \times b_{2} \times t_{2}=1 \times 0.02 \times 0.015$ (in m), Support locations: $x_{r}=0.5 L_{1}$ and $y_{r}=0.5 L_{2}$. 
Figure 23 shows the variation of maximum stretching or membrane force of the two beams with load and the stretching force distribution along the two beams for the maximum load, respectively, under the same loading pattern and geometry as that of Figure 22. In accordance with the previous figures, the stretching forces in Beam-1 show higher values compared with those of Beam- 2 .

\section{CONCLUSIONS}

In the present paper, large displacement static analysis of a crossbeam structure is presented using an energy method and variational formulation. An iterative method based on the reaction force between the two beams of the system is developed to obtain the solutions for static equilibrium case. An experimental setup is prepared and experiments are performed for concentrated loading scenarios. Although the experimental results have the same nature as those generated through the present analyses, the actual displacement values are higher than the theoretical results. This may be attributed to insufficiency in replicating stretching boundary conditions of the system. 2-D and 3-D simulation studies are also carried out using ANSYS v11.0, in order to validate the present method and both sets of results show good agreement with the results from the present analyses. The system response is studied for variation of thickness of the constituent beams and changes in position of one of the beams. The results are presented in terms of reaction force-load and displacement-load plots in dimensional form, followed by the deflected shapes of the system at static equilibrium conditions, under a particular load. The results lead to conclusions that a relatively thicker Beam-2 (in comparison with Beam-1) provides a greater stiffening effect and a centrally located supporting beam (i.e., Beam-2) provides the optimum stiffening effect. Also, the effect of various loading patterns on the structure is investigated and it is found that the system response is dependent both on the relative geometry of the two beams and the nature of loading.

\section{ACKNOWLEDGEMENTS}

The first author acknowledges the research support received from AICTE, India, vide File No.: 1-10/RID/NDF/PG/(17)2008-09 Dated : 13.03.2009.

\section{REFERENCES}

Agarwal, S., Chakraborty, A. and Gopalakrishnan, S. 2006. Large deformation analysis for anisotropic and inhomogeneous beams using exact linear static solutions. Computers \& Structures, 72(1): 91-104.

Bedair, O.K. 1997. Analysis of stiffened plates under lateral loading using sequential quadratic program. Computers \& Structures, 62(1): 63-80.

Bedair, O.K. 1998. A contribution to the stability of stiffened plates under uniform compression. Computers \& Structures, 66(5): 535-570.

Brubak, L. and Hellesland, J. 2008. Strength criteria in semi-analytical, large deflection analysis of stiffened plates in local and global bending. Thin-Walled Structures, 46: 1382-1390.

Cornil, M.B., Capolungo, L., Qu, J. and Jairazbhoy, V.A. 2007. Free vibration of a beam subjected to large static deflection. Journal of Sound and Vibration, 303: 723-740. 
Deb Nath, S.K., Reaz Ahmed S. and Kim S-G. 2010. Analytical solution of a stiffened orthotropic plate using alternative displacement potential approach. Proceedings of the Institution of Mechanical Engineers, Part G: Journal of Aerospace Engineering, 224(1): 89-99.

Fernandes, G.R. 2009. A BEM formulation for linear bending analysis of plates reinforced by beams considering different materials. Engineering Analysis with Boundary Elements, 33(8-9): 1132-1140.

Kapania, R.K. and Raciti, S. 1989. Recent advances in analysis of laminated beams and plates, Part-I: Shear effects and buckling. American Institute of Aeronautics and Astronautics Journal, 27: 935-946.

Koko, T.S. and Olson, M.D. 1991. Nonlinear analysis of stiffened plates using super elements. International Journal of Numerical Methods in Engineering, 31(2): 319343.

Kong, S., Zhou, S., Nie, Z. and Wang, K. 2008. Static and dynamic analysis of micro beams based on strain gradient elasticity theory. International Journal of Engineering Science, 47: 487-498.

Marur, P.R. 2007. Analysis of thin-walled frames considering joint flexibilities. Journal of Automobile Engineering, 221: 1221-1229.

Marur, S.R. 2001. Advances in nonlinear vibration analysis of structures. Part-I. Beams. Sadhana, 26(3): 243-249.

Mukhopadhyay, M. and Mukherjee, A. 1989. Recent advances on the dynamic behavior of stiffened plates. Shock and Vibration Digest, 21(4): 6-9.

Reddy, J.N. 1979. Finite element modeling of structural vibrations: A review of recent advances. Shock and Vibration Digest, 11: 25-39.

Sapountzakis, E.J. and Katsikadelis, J.T. 2000. Elastic deformation of ribbed plates under static, transverse and in-plane loading. Computers \& Structures, 74: 571581 .

Sapountzakis, E.J. and Mokos, V.G. 2008. Elastic deformation of ribbed plates under static, transverse and in-plane loading. Computers \& Structures, 86: 2166-2181.

Sathyamoorthy, M. 1982a. Nonlinear analysis of beams, Part-I: A survey of recent advances. Shock and Vibration Digest, 14: 19-35.

Sathyamoorthy, M. 1982b. Nonlinear Nonlinear analysis of beams, Part-II: Finiteelement methods. Shock and Vibration Digest, 14: 7-18.

Sheikh, A.H. and Mukhopadhyay, M. 2000. Geometric nonlinear analysis of stiffened plates by spline finite strip method. Computers \& Structures, 76(3): 765-785.

Turvey, G.J. and Salehi, M. 2008. Elasto-plastic large deflection response of pressure loaded circular plates stiffened by a single diametral stiffener. Thin-Walled Structures, 46: 991-1002.

Wutzow, W.W. and Paiva, J.B. 2008. Analysis of stiffened plates by the boundary element method. Engineering Analysis with Boundary Elements, 32(1): 1-10. 